\title{
A Coupling Method of New EMFE and FE for Fourth-Order Partial Differential Equation of Parabolic Type
}

\author{
Yang Liu, ${ }^{1}$ Hong Li, ${ }^{1}$ Zhichao Fang, ${ }^{1}$ Siriguleng He, ${ }^{1}$ and Jinfeng Wang ${ }^{2}$ \\ ${ }^{1}$ School of Mathematical Sciences, Inner Mongolia University, Hohhot 010021, China \\ ${ }^{2}$ School of Statistics and Mathematics, Inner Mongolia University of Finance and Economics, Hohhot 010070, China \\ Correspondence should be addressed to Yang Liu; mathliuyang@yahoo.cn and Hong Li; smslh@imu.edu.cn
}

Received 29 November 2012; Accepted 11 March 2013

Academic Editor: B. G. Konopelchenko

Copyright (c) 2013 Yang Liu et al. This is an open access article distributed under the Creative Commons Attribution License, which permits unrestricted use, distribution, and reproduction in any medium, provided the original work is properly cited.

\begin{abstract}
We propose and analyze a new numerical method, called a coupling method based on a new expanded mixed finite element (EMFE) and finite element (FE), for fourth-order partial differential equation of parabolic type. We first reduce the fourth-order parabolic equation to a coupled system of second-order equations and then solve a second-order equation by FE method and approximate the other one by a new EMFE method. We find that the new EMFE method's gradient belongs to the simple square integrable $\left(L^{2}(\Omega)\right)^{2}$ space, which avoids the use of the classical $\mathbf{H}(\operatorname{div} ; \Omega)$ space and reduces the regularity requirement on the gradient solution $\lambda=\nabla u$. For a priori error estimates based on both semidiscrete and fully discrete schemes, we introduce a new expanded mixed projection and some important lemmas. We derive the optimal a priori error estimates in $L^{2}$ and $H^{1}$-norm for both the scalar unknown $u$ and the diffusion term $\gamma$ and a priori error estimates in $\left(L^{2}\right)^{2}$-norm for its gradient $\lambda$ and its flux $\boldsymbol{\sigma}$ (the coefficients times the negative gradient). Finally, we provide some numerical results to illustrate the efficiency of our method.
\end{abstract}

\section{Introduction}

In recent years, many researchers have studied some numerical methods for fourth-order elliptic equations [1-6], fourthorder parabolic equations [5-9], fourth-order wave equations $[10,11]$, and so on. Chen [1] proposed and analyzed an expanded mixed finite element method for fourth-order elliptic problems. In [2], Chen et al. studied an anisotropic nonconforming element for fourth-order elliptic singular perturbation problem. In [3-6], some mixed finite element (MFE) methods were studied for fourth-order linear/nonlinear elliptic equations. In [7], the FE method was studied for nonlinear Cahn-Hilliard equation. The optimalorder error estimates were obtained in $L^{2}$-norm by means of an FE biharmonic projection approximation. In [12], the MFE methods were studied for solving a fourth-order nonlinear reaction diffusion equation. In [8], an $H^{1}$-Galerkin MFE method was studied for solving the fourth-order parabolic partial differential equations. Optimal error estimates were derived for both semidiscrete and fully discrete schemes for problems in one space dimension, and error estimates were derived for semidiscrete scheme for several space dimensions, and the stability for fully discrete scheme was proved by the iteration method. In [9], the FE method was studied for fourth-order nonlinear parabolic equation. He et al. [10] studied and analyzed the explicit/implicit MFE methods for a class of fourth-order wave equations. Shi and Peng [13] studied the finite element methods for fourth-order eigenvalue problems on anisotropic meshes. Liu et al. [11] studied a $C^{1}$-conforming FE method for nonlinear fourthorder wave equation. In this paper, we consider the following fourth-order partial differential equations of parabolic type:

$$
\begin{gathered}
u_{t}+\nabla \cdot(b(\mathbf{x}, t) \nabla(\nabla \cdot(a(t) \nabla u)))=f(\mathbf{x}, t), \quad(\mathbf{x}, t) \in \Omega \times J, \\
u(\mathbf{x}, t)=\Delta u(\mathbf{x}, t)=0, \quad(\mathbf{x}, t) \in \partial \Omega \times \bar{J}, \\
u(\mathbf{x}, 0)=u_{0}(\mathbf{x}), \quad \mathbf{x} \in \Omega,
\end{gathered}
$$


where $\Omega \subset R^{2}$ is a bounded convex polygonal domain with the Lipschitz continuous boundary $\partial \Omega$ and $J=(0, T]$ is the time interval with $0<T<\infty$. The initial value $u_{0}(\mathbf{x})$ and $f(\mathbf{x}, t)$ are given functions; coefficients $a=a(t)$ and $b=b(\mathbf{x}, t)$ satisfy

$$
\begin{aligned}
& \mathbf{A}_{1}: 0<a_{0} \leq a(t) \leq a_{1}<+\infty, \quad\left|a_{t}(t)\right| \leq c_{0}, \\
& \mathbf{A}_{2}: 0<b_{0} \leq b(\mathbf{x}, t) \leq b_{1}<+\infty, \quad\left|b_{t}(\mathbf{x}, t)\right| \leq c_{0},
\end{aligned}
$$

for some positive constants $a_{0}, a_{1}, b_{0}, b_{1}$, and $c_{0}$.

Chen proposed the EMFE method for second-order linear/quasilinear elliptic equation [14-16] and fourth-order linear elliptic equation [1]. Then the method was applied to other partial differential equations $[17,18]$. Compared to standard MFE methods, the EMFE method is expanded in the sense that three variables are explicitly approximated, namely, the scalar unknown, its gradient, and its flux. In recent years, many numerical method based on the expanded mixed scheme, such as two-grid EMFE methods [19-22], $H^{1}$ Galerkin EMFE methods [23-25], expanded mixed covolume method [26], expanded mixed hybrid method [27], and positive definite EMFE method [28], have been proposed and discussed.

In [29], we developed and analyzed a new EMFE method for second-order elliptic problems based on the mixed weak formulation [30-32]. The new EMFE method [29], whose gradient belongs to the square integrable $\left(L^{2}(\Omega)\right)^{2}$ space instead of the classical $\mathbf{H}(\operatorname{div} ; \Omega)$ space, is different from Chen's EMFE method [14-16].

In this paper, we present a new coupling method of new EMFE scheme [29] and FE scheme for fourth-order partial differential equation of parabolic type. We first introduce an auxiliary variable $\gamma=-\nabla \cdot(a(t) \nabla u)$ to reduce the fourthorder parabolic equation to a coupled system of second-order equations, then solve a second-order equation by FE method. For the other one, we introduce the two auxiliary variables, $\lambda=\nabla u$ and $\sigma=-a(t) \nabla u=-a \lambda$, to split the equation into a first-order system of equations, then approximate that by a new EMFE method. Because the new EMFE method's gradient belongs to the simple square integrable $\left(L^{2}(\Omega)\right)^{2}$ space, the regularity requirement on the gradient solution $\lambda=\nabla u$ is reduced. We apply the new coupling method of new EMFE and FE to derive a priori error estimates based on both semidiscrete and fully discrete schemes; that is to say, we obtain the optimal a priori error estimates in $L^{2}$ and $H^{1}$ norm for both the scalar unknown $u$ and the diffusion term $\gamma$ and a priori error estimates in $\left(L^{2}\right)^{2}$-norm for its gradient $\lambda$ and its flux $\boldsymbol{\sigma}$. Finally, we provide some numerical results to verify our theoretical analysis.

Throughout this paper, $C$ will denote a generic positive constant which does not depend on the spatial mesh parameter $h$ or the time step $\Delta t$. At the same time, we denote the natural inner product in $L^{2}(\Omega)$ or $\left(L^{2}(\Omega)\right)^{2}$ by $(\cdot, \cdot)$ with the corresponding norm $\|\cdot\|$. The other notations and definitions for the Sobolev spaces as in $[33,34]$ are used.

\section{New Expanded Mixed Scheme}

We first introduce an auxiliary variable $\gamma=-\nabla \cdot(a(t) \nabla u)$ to obtain the following coupled system of second-order equations:

$$
\begin{gathered}
u_{t}-\nabla \cdot(b(\mathbf{x}, t) \nabla \gamma)=f(\mathbf{x}, t), \\
\gamma+\nabla \cdot(a(t) \nabla u)=0, \\
u(\mathbf{x}, t)=\gamma(\mathbf{x}, t)=0 .
\end{gathered}
$$

For (3b), we introduce the two auxiliary variables, $\lambda=\nabla u$ and $\boldsymbol{\sigma}=-a(t) \nabla u=-a \boldsymbol{\lambda}$, to obtain the following first-order system of equations:

$$
\begin{gathered}
u_{t}-\nabla \cdot(b \nabla \gamma)=f(\mathbf{x}, t), \\
\gamma-\nabla \cdot \boldsymbol{\sigma}=0, \\
\boldsymbol{\sigma}+a \boldsymbol{\lambda}=0, \\
\lambda-\nabla u=0, \\
u(\mathbf{x}, t)=\gamma(\mathbf{x}, t)=0 .
\end{gathered}
$$

Using Green's formula, the new mixed weak formulation of (4a)-(4e) is to determine $\{u, \gamma, \lambda, \sigma\}:[0, T] \mapsto H_{0}^{1}(\Omega) \times$ $H_{0}^{1}(\Omega) \times\left(L^{2}(\Omega)\right)^{2} \times\left(L^{2}(\Omega)\right)^{2}$ such that

$$
\begin{gathered}
\left(u_{t}, v\right)+(b \nabla \gamma, \nabla v)=(f, v), \quad \forall v \in H_{0}^{1}(\Omega), \\
(\gamma, g)+(\boldsymbol{\sigma}, \nabla g)=0, \quad \forall g \in H_{0}^{1}(\Omega), \\
(\boldsymbol{\sigma}, \mathbf{z})+(a \boldsymbol{\lambda}, \mathbf{z})=0, \quad \forall \mathbf{z} \in\left(L^{2}(\Omega)\right)^{2}, \\
(\lambda, \mathbf{w})-(\nabla u, \mathbf{w})=0, \quad \forall \mathbf{w} \in\left(L^{2}(\Omega)\right)^{2} .
\end{gathered}
$$

Then, the semidiscrete coupled EMFE scheme-FE scheme for (5a)-(5d) is to find $\left\{u_{h}, \gamma_{h}, \lambda_{h}, \boldsymbol{\sigma}_{h}\right\}:[0, T] \mapsto V_{h} \times V_{h} \times \mathbf{W}_{h} \times$ $\mathbf{W}_{h}$ such that

$$
\begin{array}{cc}
\left(u_{h t}, v_{h}\right)+\left(b \nabla \gamma_{h}, \nabla v_{h}\right)=\left(f, v_{h}\right), & \forall v_{h} \in V_{h}, \\
\left(\gamma_{h}, g_{h}\right)+\left(\sigma_{h}, \nabla g_{h}\right)=0, & \forall g_{h} \in V_{h}, \\
\left(\sigma_{h}, \mathbf{z}_{h}\right)+\left(a \boldsymbol{\lambda}_{h}, \mathbf{z}_{h}\right)=0, & \forall \mathbf{z}_{h} \in \mathbf{W}_{h}, \\
\left(\boldsymbol{\lambda}_{h}, \mathbf{w}_{h}\right)-\left(\nabla u_{h}, \mathbf{w}_{h}\right)=0, & \forall \mathbf{w}_{h} \in \mathbf{W}_{h} .
\end{array}
$$

By the theory of differential equations [35], we can prove the existence and uniqueness of solution for semidiscrete scheme (6a)-(6d).

Remark 1. From the new coupling weak formulation (5a)(5d), we can find that the gradient belongs to the weaker square integrable $\left(L^{2}(\Omega)\right)^{2}$ space taking the place of the classical $\mathbf{H}(\operatorname{div} ; \Omega)$ space. It is easy to see that our method reduces the regularity requirement on the gradient solution $\lambda=\nabla u$. 
Remark 2. In the semidiscrete coupling scheme (6a)-(6d), the MFE space $\left(V_{h}, \mathbf{W}_{h}\right)$ based on the FE pair $P_{1}-P_{0}^{2}$ is chosen as follows:

$$
\begin{aligned}
& V_{h}=\left\{v_{h} \in C^{0}(\Omega) \cap H_{0}^{1}(\Omega) \mid v_{h} \in P_{1}(K), \forall K \in \mathscr{K}_{h}\right\}, \\
& \mathbf{W}_{h}=\left\{\mathbf{w}_{h}=\left(w_{1 h}, w_{2 h}\right) \in\left(L^{2}(\Omega)\right)^{2} \mid\right. \\
&\left.w_{1 h}, w_{2 h} \in P_{0}(K), \forall K \in \mathscr{K}_{h}\right\} .
\end{aligned}
$$

From [30, 31], we know that MFE space $\left(V_{h}, \mathbf{W}_{h}\right)$ satisfies the discrete LBB condition.

Remark 3. From (6a)-(6d), we know that (6a) is an FE scheme for $u_{h}, \gamma_{h}, v_{h} \in V_{h}$ and that (6b)-(6d) is a new EMFE scheme for $\left(\gamma_{h}, \boldsymbol{\sigma}_{h}, \boldsymbol{\lambda}_{h}\right),\left(g_{h}, \mathbf{z}_{h}, \mathbf{w}_{h}\right) \in V_{h} \times \mathbf{W}_{h} \times \mathbf{W}_{h}$. So the scheme (6a)-(6d) is called as the coupling system of new EMFE scheme and FE scheme.

Remark 4. The finite element spaces $V_{h}$ and $\mathbf{W}_{h}$ for the new EMFE method are chosen as finite dimensional subspaces of $H_{0}^{1}(\Omega)$ and $\left(L^{2}(\Omega)\right)^{2}$, respectively. However, $V_{h}$ and $\mathbf{W}_{h}$ for $H^{1}$-Galerkin MFE method in two space variables in [8] are finite dimensional subspaces of $H_{0}^{1}(\Omega)$ and $\mathbf{H}(\operatorname{div} ; \Omega)$, respectively. Compared to the complex $\mathbf{H}(\operatorname{div} ; \Omega)$ space used in the $H^{1}$-Galerkin MFE method, we use the weaker $\left(L^{2}(\Omega)\right)^{2}$ space for our EMFE method.

\section{Semidiscrete Error Estimates}

For deriving some a priori error estimates of our method, we first introduce the projection operator $\mathscr{P}_{h}$ and the new expanded mixed elliptic projection operator $\mathscr{R}_{h}$ associated with the coupled equations.

Lemma 5. One now defines a linear projection operator $\mathscr{P}_{h}$ : $H_{0}^{1}(\Omega) \rightarrow V_{h}$ as

$$
\left(b \nabla\left(\gamma-\mathscr{P}_{h} \gamma\right), \nabla v_{h}\right)=0, \quad \forall v_{h} \in V_{h},
$$

and then

$$
\begin{gathered}
\left\|\gamma-\mathscr{P}_{h} \gamma\right\|_{L^{2}(\Omega)}+h\left\|\gamma-\mathscr{P}_{h} \gamma\right\|_{H^{1}} \leq C h^{2}\|\gamma\|_{H^{2}}, \\
\left\|\gamma_{t}-\mathscr{P}_{h} \gamma_{t}\right\|_{L^{2}(\Omega)} \leq C h^{2}\left(\|\gamma\|_{H^{2}}+\left\|\gamma_{t}\right\|_{H^{2}}\right), \\
\left\|\gamma_{t t}-\mathscr{P}_{h} \gamma_{t t}\right\|_{L^{2}(\Omega)} \leq C h^{2}\left(\|\gamma\|_{H^{2}}+\left\|\gamma_{t}\right\|_{H^{2}}+\left\|\gamma_{t t}\right\|_{H^{2}}\right) .
\end{gathered}
$$

Let $\left(\mathscr{R}_{h} \mathcal{u}, \mathscr{R}_{h} \boldsymbol{\lambda}, \mathscr{R}_{h} \boldsymbol{\sigma}\right):[0, T] \rightarrow V_{h} \times \mathbf{W}_{h} \times \mathbf{W}_{h}$ be given by the following new expanded mixed relations [29]

$$
\begin{gathered}
\left(\boldsymbol{\sigma}-\mathscr{R}_{h} \boldsymbol{\sigma}, \nabla g_{h}\right)=0, \quad \forall g_{h} \in V_{h}, \\
\left(\boldsymbol{\sigma}-\mathscr{R}_{h} \boldsymbol{\sigma}, \mathbf{z}_{h}\right)+\left(a\left(\boldsymbol{\lambda}-\mathscr{R}_{h} \boldsymbol{\lambda}\right), \mathbf{z}_{h}\right)=0, \quad \forall \mathbf{z}_{h} \in \mathbf{W}_{h}, \\
\left(\boldsymbol{\lambda}-\mathscr{R}_{h} \boldsymbol{\lambda}, \mathbf{w}_{h}\right)-\left(\nabla\left(u-\mathscr{R}_{h} u\right), \mathbf{w}_{h}\right)=0, \quad \forall \mathbf{w}_{h} \in \mathbf{W}_{h} .
\end{gathered}
$$

Then the following two important lemmas based on the new expanded mixed projection (10a)-(10c) hold.

Lemma 6. There is a constant $C>0$ independent of the spatial mesh parameter $h$ such that

$$
\begin{gathered}
\left\|\lambda-\mathscr{R}_{h} \lambda\right\| \leq \operatorname{Ch}\left(\|\lambda\|_{\left(H^{1}\right)^{2}}+\|u\|_{H^{2}}\right), \\
\left\|\boldsymbol{\sigma}-\mathscr{R}_{h} \boldsymbol{\sigma}\right\| \leq \operatorname{Ch}\left(\|u\|_{H^{2}}+\|\boldsymbol{\sigma}\|_{\left(H^{1}\right)^{2}}+\|\lambda\|_{\left(H^{1}\right)^{2}}\right), \\
\left\|\nabla\left(u-\mathscr{R}_{h} u\right)\right\| \leq \operatorname{Ch}\left(\|u\|_{H^{2}}+\|\lambda\|_{\left(H^{1}\right)^{2}}\right) .
\end{gathered}
$$

Lemma 7. There is a constant $C>0$ independent of the spatial mesh parameter $h$ such that

$$
\begin{gathered}
\left\|u-\mathscr{R}_{h} u\right\| \leq C h^{2}\|u\|_{H^{2}}, \\
\left\|u_{t}-\mathscr{R}_{h} u_{t}\right\| \leq C h^{2}\left\|u_{t}\right\|_{H^{2}}, \\
\left\|u_{t t}-\mathscr{R}_{h} u_{t t}\right\| \leq C h^{2}\left\|u_{t t}\right\|_{H^{2}}, \\
\left\|u-\mathscr{R}_{h} u\right\|_{1} \leq C h\left(\|u\|_{H^{2}}+\|\lambda\|_{\left(H^{1}\right)^{2}}\right) .
\end{gathered}
$$

In [29], we can obtain the detailed proof for Lemmas 6-7.

For a priori error estimates, we write the errors as

$$
\begin{aligned}
& \gamma-\gamma_{h}=\gamma-\mathscr{P}_{h} \gamma+\mathscr{P}_{h} \gamma-\gamma_{h}=\psi+\Phi ; \\
& u-u_{h}=u-\mathscr{R}_{h} u+\mathscr{R}_{h} u-u_{h}=\eta+\varsigma ; \\
& \lambda-\lambda_{h}=\boldsymbol{\lambda}-\mathscr{R}_{h} \boldsymbol{\lambda}+\mathscr{R}_{h} \boldsymbol{\lambda}-\boldsymbol{\lambda}_{h}=\boldsymbol{\delta}+\boldsymbol{\theta} ; \\
& \boldsymbol{\sigma}-\boldsymbol{\sigma}_{h}=\boldsymbol{\sigma}-\mathscr{R}_{h} \boldsymbol{\sigma}+\mathscr{R}_{h} \boldsymbol{\sigma}-\boldsymbol{\sigma}_{h}=\boldsymbol{\rho}+\boldsymbol{\xi} .
\end{aligned}
$$

Using (5a), (5b), (5c), (5d), (6a), (6b), (6c), (6d), (8), (10a), $(10 b)$, and (10c) we can obtain the error equations

$$
\begin{gathered}
\left(\varsigma_{t}, v_{h}\right)+\left(b \nabla \omega, \nabla v_{h}\right)=-\left(\eta_{t}, v_{h}\right), \quad \forall v_{h} \in V_{h}, \\
\left(\varrho, g_{h}\right)+\left(\xi, \nabla g_{h}\right)=-\left(\psi, g_{h}\right), \quad \forall g_{h} \in V_{h}, \\
\left(\xi, \mathbf{z}_{h}\right)+\left(a \boldsymbol{\theta}, \mathbf{z}_{h}\right)=0, \quad \forall \mathbf{z}_{h} \in \mathbf{W}_{h}, \\
\left(\boldsymbol{\theta}, \mathbf{w}_{h}\right)-\left(\nabla \varsigma, \mathbf{w}_{h}\right)=0, \quad \forall \mathbf{w}_{h} \in \mathbf{W}_{h} .
\end{gathered}
$$

We will prove the error estimates for semidiscrete scheme.

Theorem 8. Suppose that $\gamma, \gamma_{t}, u_{t} \in L^{2}\left(H^{2}(\Omega)\right), \lambda, \sigma \in$ $L^{\infty}\left(\left(H^{1}(\Omega)\right)^{2}\right)$, and $u, \gamma \in L^{\infty}\left(H^{2}(\Omega)\right)$; then there exists a constant $C>0$ independent of the spatial mesh parameter $h$ such that

$$
\begin{array}{r}
\left\|u-u_{h}\right\| \leq C h^{2}\left[\|u\|_{L^{\infty}\left(H^{2}\right)}+\|\gamma\|_{L^{\infty}\left(H^{2}\right)}\right. \\
\left.+\left(\int_{0}^{t}\|\| \mathbf{\square} \|^{2} d s\right)^{1 / 2}\right],
\end{array}
$$




$$
\begin{aligned}
& \left\|u-u_{h}\right\|_{1} \leq C h\left[\|\lambda\|_{L^{\infty}\left(\left(H^{1}\right)^{2}\right)}+\|u\|_{L^{\infty}\left(H^{2}\right)}\right. \\
& \left.+\|\gamma\|_{L^{\infty}\left(H^{2}\right)}+\left(\int_{0}^{t}\|\| \mathbf{|}\|\|^{2} d s\right)^{1 / 2}\right] \\
& \left\|\boldsymbol{\lambda}-\boldsymbol{\lambda}_{h}\right\| \leq C h\left[\|\boldsymbol{\lambda}\|_{L^{\infty}\left(\left(H^{1}\right)^{2}\right)}+\|\gamma\|_{L^{\infty}\left(H^{2}\right)}\right. \\
& \left.+\left(\int_{0}^{t}\|\| \mathbf{|}\|\|^{2} d s\right)^{1 / 2}\right] \\
& \left\|\boldsymbol{\sigma}-\boldsymbol{\sigma}_{h}\right\| \leq C h\left[\|u\|_{L^{\infty}\left(H^{2}\right)}+\|\boldsymbol{\sigma}\|_{L^{\infty}\left(\left(H^{1}\right)^{2}\right)}+\|\lambda\|_{L^{\infty}\left(\left(H^{1}\right)^{2}\right)}\right. \\
& \left.+\|\gamma\|_{L^{\infty}\left(H^{2}\right)}+\left(\int_{0}^{t}\|\| \|^{2} d s\right)^{1 / 2}\right]
\end{aligned}
$$

where $\||\mathbf{|}|\|^{2} \triangleq\left\|u_{t}\right\|_{H^{2}}^{2}+\|\gamma\|_{H^{2}}^{2}+\left\|\gamma_{t}\right\|_{H^{2}}^{2}$.

Proof. Choose $v_{h}=\omega, g_{h}=\varsigma_{t}, \mathbf{z}_{h}=\nabla \varsigma_{t}$, and $\mathbf{w}_{h}=\nabla \varsigma_{t}$ in (14a)-(14d) to obtain

$$
\begin{gathered}
\left(\varsigma_{t}, \omega\right)+\left\|b^{1 / 2} \nabla \omega\right\|^{2}=-\left(\eta_{t}, \omega\right), \\
-\left(\omega, \varsigma_{t}\right)-\left(\xi, \nabla \varsigma_{t}\right)=\left(\psi, \varsigma_{t}\right), \\
\left(\xi, \nabla \varsigma_{t}\right)+\left(a \boldsymbol{\theta}, \nabla \varsigma_{t}\right)=0, \\
-\left(a \boldsymbol{\theta}, \nabla \varsigma_{t}\right)+\frac{1}{2} \frac{d}{d t}\left\|a^{1 / 2} \nabla \varsigma\right\|^{2}=\frac{1}{2}\left(a_{t} \nabla \varsigma, \nabla \varsigma\right) .
\end{gathered}
$$

Adding the above four equations and using Cauchy-Schwarz inequality and Young's inequality, we have

$$
\begin{aligned}
& \frac{1}{2} \frac{d}{d t}\left\|a^{1 / 2} \nabla \varsigma\right\|^{2}+\left\|b^{1 / 2} \nabla \omega\right\|^{2} \\
&=-\left(\eta_{t}, \omega\right)+\left(\psi, \varsigma_{t}\right)+\frac{1}{2}\left(a_{t} \nabla \varsigma, \nabla \varsigma\right) \\
&=-\left(\eta_{t}, \omega\right)+\frac{d}{d t}(\psi, \varsigma)-\left(\psi_{t}, \varsigma\right)+\frac{1}{2}\left(a_{t} \nabla \varsigma, \nabla \varsigma\right) \\
& \leq \frac{d}{d t}(\psi, \varsigma)+\left\|\eta_{t}\right\|\|\omega\|+C\left(\|\varsigma\|_{1}^{2}+\left\|\psi_{t}\right\|^{2}\right) .
\end{aligned}
$$

Integrate with respect to time from 0 to $t$ to obtain

$$
\begin{aligned}
& a_{0}\|\nabla \varsigma\|^{2}+2 b_{0} \int_{0}^{t}\|\nabla \omega\|^{2} d s \\
& \quad \leq 2(\psi, \varsigma)+2 \int_{0}^{t}\left\|\eta_{t}\right\|\|\omega\| d s+C \int_{0}^{t}\left(\|\varsigma\|_{1}^{2}+\left\|\psi_{t}\right\|^{2}\right) d s .
\end{aligned}
$$

Noting that $\varsigma, \varpi \in H_{0}^{1}(\Omega)$, we use Poincaré inequality to have

$$
\|\varsigma\|_{1} \leq C_{1}\|\nabla \varsigma\|, \quad\|\omega\| \leq C_{2}\|\nabla \omega\| .
$$

Using (18), (19), Cauchy-Schwarz inequality and Young inequality, we have

$$
\begin{aligned}
a_{0}\|\nabla \varsigma\|^{2} & +2 b_{0} \int_{0}^{t}\|\nabla \omega\|^{2} d s \\
\leq & 2\|\psi\|\|\varsigma\|+2 \int_{0}^{t}\left\|\eta_{t}\right\|\|\omega\| d s+C \int_{0}^{t}\left(\|\varsigma\|_{1}^{2}+\left\|\psi_{t}\right\|^{2}\right) d s \\
\leq & 2 C_{1}\|\psi\|\|\nabla \varsigma\|+2 C_{2} \int_{0}^{t}\left\|\eta_{t}\right\|\|\nabla \omega\| d s \\
& +C \int_{0}^{t}\left(C_{1}^{2}\|\nabla \varsigma\|^{2}+\left\|\psi_{t}\right\|^{2}\right) d s \\
\leq & \frac{8 C_{1}^{2}}{a_{0}}\|\psi\|^{2}+\frac{a_{0}}{2}\|\nabla \varsigma\|^{2}+\frac{4 C_{2}^{2}}{b_{0}} \int_{0}^{t}\left\|\eta_{t}\right\|^{2} d s \\
& +b_{0} \int_{0}^{t}\|\nabla \omega\|^{2} d s \\
& +C \int_{0}^{t}\left(C_{1}^{2}\|\nabla \varsigma\|^{2}+\left\|\psi_{t}\right\|^{2}\right) d s .
\end{aligned}
$$

Using Gronwall's lemma, we get

$$
\begin{aligned}
\|\nabla \varsigma\|^{2} & +\int_{0}^{t}\|\nabla \omega\|^{2} d s \\
& \leq C\left(\|\psi\|^{2}+\int_{0}^{t}\left(\left\|\eta_{t}\right\|^{2}+\|\psi\|^{2}+\left\|\psi_{t}\right\|^{2}\right) d s\right) .
\end{aligned}
$$

Substitute (19) into (21) to have

$$
\begin{aligned}
\|\varsigma\|^{2} & +\int_{0}^{t}\|\omega\|^{2} d s \\
& \leq C\left(\|\psi\|^{2}+\int_{0}^{t}\left(\left\|\eta_{t}\right\|^{2}+\|\psi\|^{2}+\left\|\psi_{t}\right\|^{2}\right) d s\right) .
\end{aligned}
$$

Taking $\mathbf{w}_{h}=\boldsymbol{\theta}$ in (14d) and using (22), we get

$$
\|\boldsymbol{\theta}\|^{2} \leq C\left(\|\psi\|^{2}+\int_{0}^{t}\left(\left\|\eta_{t}\right\|^{2}+\|\psi\|^{2}+\left\|\psi_{t}\right\|^{2}\right) d s\right) .
$$

Choose $\mathbf{z}_{h}=\boldsymbol{\xi}$ in (14c) and use (22) to obtain

$$
\|\xi\|^{2} \leq C\left(\|\psi\|^{2}+\int_{0}^{t}\left(\left\|\eta_{t}\right\|^{2}+\|\psi\|^{2}+\left\|\psi_{t}\right\|^{2}\right) d s\right) .
$$

Combining Lemmas 5-7, (21)-(24) and using the triangle inequality, we get the error estimates for Theorem 8 .

Remark 9. From Theorem 8, we can see that

$$
\left\|u-u_{h}\right\|_{L^{\infty}\left(L^{2}\right)}+h\left\|u-u_{h}\right\|_{L^{\infty}\left(H^{1}\right)}=O\left(h^{2}\right) ;
$$

that is to say, a priori error estimates in $L^{2}$ and $H^{1}$-norms for the scalar unknown $u$ are optimal. 
Theorem 10. Suppose that $\partial^{j} u / \partial t^{j}, \partial^{j} \gamma / \partial t^{j} \in L^{2}\left(H^{2}(\Omega)\right)$ and $\gamma, \gamma_{t}, u_{t} \in L^{\infty}\left(H^{2}(\Omega)\right)$; then there exists a constant $C>0$ independent of the spatial mesh parameter $h$ such that

$$
\begin{aligned}
\left\|\gamma-\gamma_{h}\right\|+h\left\|\gamma-\gamma_{h}\right\|_{1} & \\
\leq C h^{2} & {\left[\left\|u_{t}\right\|_{L^{\infty}\left(H^{2}\right)}+\|\gamma\|_{L^{\infty}\left(H^{2}\right)}+\left\|\gamma_{t}\right\|_{L^{\infty}\left(H^{2}\right)}\right.} \\
& \left.+\left(\int_{0}^{t}\left(\sum_{j=0}^{2}\left\|\frac{\partial^{j} u}{\partial t^{j}}\right\|_{H^{2}}^{2}+\left\|\frac{\partial^{j} \gamma}{\partial t^{j}}\right\|_{H^{2}}^{2}\right) d s\right)^{1 / 2}\right] .
\end{aligned}
$$

Proof. Differentiating (14b), (14c), and (14d) with respect to time $t$, respectively, we get

$$
\begin{gathered}
\left(\varsigma_{t}, v_{h}\right)+\left(b \nabla \omega, \nabla v_{h}\right)=-\left(\eta_{t}, v_{h}\right), \\
\left(\varrho_{t}, g_{h}\right)+\left(\boldsymbol{\xi}_{t}, \nabla g_{h}\right)=-\left(\psi_{t}, g_{h}\right), \\
\left(\boldsymbol{\xi}_{t}, \mathbf{z}_{h}\right)+\left(a_{t} \boldsymbol{\theta}, \mathbf{z}_{h}\right)+\left(a \boldsymbol{\theta}_{t}, \mathbf{z}_{h}\right)=0, \\
\left(\boldsymbol{\theta}_{t}, \mathbf{w}_{h}\right)-\left(\nabla \varsigma_{t}, \mathbf{w}_{h}\right)=0 .
\end{gathered}
$$

Choose $v_{h}=\omega_{t}, g_{h}=\varsigma_{t}, \mathbf{z}_{h}=\nabla \varsigma_{t}$, and $\mathbf{w}_{h}=\nabla \varsigma_{t}$ in (27a)(27d) to get

$$
\begin{aligned}
&\left(\varsigma_{t}, \omega_{t}\right)+\frac{1}{2} \frac{d}{d t}\left\|b^{1 / 2} \nabla \omega\right\|^{2}=-\left(\eta_{t}, \omega_{t}\right)+\frac{1}{2}\left(b_{t} \nabla \omega, \nabla \omega\right) \\
&-\left(\varrho_{t}, \varsigma_{t}\right)-\left(\xi_{t}, \nabla \varsigma_{t}\right)=\left(\psi_{t}, \varsigma_{t}\right) \\
&\left(\xi_{t}, \nabla \varsigma_{t}\right)+\left(a_{t} \boldsymbol{\theta}, \nabla \varsigma_{t}\right)+\left(a \boldsymbol{\theta}_{t}, \nabla \varsigma_{t}\right)=0 \\
&-\left(a \boldsymbol{\theta}_{t}, \nabla \varsigma_{t}\right)+\left\|a^{1 / 2} \nabla \varsigma_{t}\right\|^{2}=0
\end{aligned}
$$

Adding the four equations for (28a)-(28d), we have

$$
\begin{aligned}
\frac{1}{2} \frac{d}{d t} \| & b^{1 / 2} \nabla \omega\left\|^{2}+\right\| a^{1 / 2} \nabla \varsigma_{t} \|^{2} \\
= & -\left(\eta_{t}, \omega_{t}\right)+\left(\psi_{t}, \varsigma_{t}\right)-\left(a_{t} \boldsymbol{\theta}, \nabla \varsigma_{t}\right)+\frac{1}{2}\left(b_{t} \nabla \omega, \nabla \omega\right) \\
= & -\frac{d}{d t}\left(\eta_{t}, \omega\right)+\left(\eta_{t t}, \omega\right)+\frac{d}{d t}\left(\psi_{t}, \varsigma\right) \\
& -\left(\psi_{t t}, \varsigma\right)-\left(a_{t} \boldsymbol{\theta}, \nabla \varsigma_{t}\right)+\frac{1}{2}\left(b_{t} \nabla \omega, \nabla \omega\right) .
\end{aligned}
$$

Integrate with respect to time from 0 to $t$ and use (19), Cauchy-Schwarz inequality, and Young inequality to get

$$
\begin{aligned}
b_{0}\|\nabla \omega\|^{2} & +2 a_{0} \int_{0}^{t}\left\|\nabla \varsigma_{t}\right\|^{2} d s \\
\leq & -2\left(\eta_{t}, \varrho\right)+2 C_{2} \int_{0}^{t}\left\|\eta_{t t}\right\|\|\nabla \omega\| d s+2\left(\psi_{t}, \varsigma\right) \\
& +C \int_{0}^{t}\left(\left\|\psi_{t t}\right\|^{2}+\|\varsigma\|^{2}+\|\boldsymbol{\theta}\|^{2}\right) d s+a_{0} \int_{0}^{t}\left\|\nabla \varsigma_{t}\right\|^{2} d s \\
\leq & \frac{8 C_{2}^{2}}{b_{0}}\left\|\eta_{t}\right\|^{2}+\frac{b_{0}}{2}\|\nabla \omega\|^{2}+\left\|\psi_{t}\right\|^{2}+\|\varsigma\|^{2}+a_{0} \int_{0}^{t}\left\|\nabla \varsigma_{t}\right\|^{2} d s \\
& +C \int_{0}^{t}\left(\left\|\psi_{t t}\right\|^{2}+\|\varsigma\|^{2}+\|\boldsymbol{\theta}\|^{2}+\left\|\eta_{t t}\right\|^{2}+\|\nabla \omega\|^{2}\right) d s .
\end{aligned}
$$

Substituting (22) and (23) into (30) and using Gronwall lemma, we get

$$
\begin{aligned}
&\|\nabla \omega\|^{2}+\int_{0}^{t}\left\|\nabla c_{t}\right\|^{2} d s \\
& \leq C\left\|\eta_{t}\right\|^{2}+\|\psi\|^{2}+\left\|\psi_{t}\right\|^{2} \\
&+C \int_{0}^{t}\left(\|\eta\|^{2}+\left\|\eta_{t}\right\|^{2}+\left\|\eta_{t t}\right\|^{2}\right. \\
&\left.+\|\psi\|^{2}+\left\|\psi_{t}\right\|^{2}+\left\|\psi_{t t}\right\|^{2}\right) d s .
\end{aligned}
$$

Use (19) to obtain

$$
\begin{aligned}
\|\Phi\|^{2} \leq & C\left\|\eta_{t}\right\|^{2}+\|\psi\|^{2}+\left\|\psi_{t}\right\|^{2} \\
+C \int_{0}^{t}( & \|\eta\|^{2}+\left\|\eta_{t}\right\|^{2}+\left\|\eta_{t t}\right\|^{2} \\
& \left.+\|\psi\|^{2}+\left\|\psi_{t}\right\|^{2}+\left\|\psi_{t t}\right\|^{2}\right) d s .
\end{aligned}
$$

Combining Lemmas 5-7, (31), and (32) and using the triangle inequality, we obtain the error estimates for Theorem 10 .

Remark 11. From Theorem 10, we can see that

$$
\left\|\gamma-\gamma_{h}\right\|_{L^{\infty}\left(L^{2}\right)}+h\left\|\gamma-\gamma_{h}\right\|_{L^{\infty}\left(H^{1}\right)}=O\left(h^{2}\right) ;
$$

that is to say, a priori error estimates in $L^{2}$ and $H^{1}$-norm for the diffusion term $\gamma$ are optimal.

\section{Fully Discrete Error Estimates}

In the following analysis, we will derive a priori error estimates based on fully discrete backward Euler scheme. Let $0=t_{0}<t_{1}<t_{2}<\cdots<t_{M}=T$ be a given partition of the time interval $[0, T]$ with step length $\Delta t=T / M$ and nodes $t_{n}=n \Delta t$, for some positive integer $M$. For a smooth function $\phi$ on $[0, T]$, define $\phi^{n}=\phi\left(t_{n}\right)$. 
For (5a), (5b), (5c), and (5d), we have the following equivalent formulation:

$\left(\frac{u^{n}-u^{n-1}}{\Delta t}, v\right)+\left(b^{n} \nabla \gamma^{n}, \nabla v\right)=\left(f^{n}+R_{1}^{n}, v\right), \quad \forall v \in H_{0}^{1}(\Omega)$,

$$
\begin{array}{cc}
\left(\gamma^{n}, g\right)+\left(\boldsymbol{\sigma}^{n}, \nabla g\right)=0, & \forall g \in H_{0}^{1}(\Omega), \\
\left(\boldsymbol{\sigma}^{n}, \mathbf{z}\right)+\left(a^{n} \lambda^{n}, \mathbf{z}\right)=0, & \forall \mathbf{z} \in\left(L^{2}(\Omega)\right)^{2}, \\
\left(\lambda^{n}, \mathbf{w}\right)-\left(\nabla u^{n}, \mathbf{w}\right)=0, & \forall \mathbf{w} \in\left(L^{2}(\Omega)\right)^{2},
\end{array}
$$

where

$$
R_{1}^{n}=\frac{u^{n}-u^{n-1}}{\Delta t}-u_{t}\left(t_{n}\right)=\frac{1}{\Delta t} \int_{t_{n-1}}^{t_{n}}\left(t_{n-1}-s\right) u_{t t} d s .
$$

Now a fully discrete procedure is to find $\left(u_{h}^{n}, \gamma_{h}^{n}, \lambda_{h}^{n}, \sigma_{h}^{n}\right) \in V_{h} \times$ $V_{h} \times \mathbf{W}_{h} \times \mathbf{W}_{h}(n=0,1, \ldots, M)$ such that

$$
\begin{gathered}
\left(\frac{u_{h}^{n}-u_{h}^{n-1}}{\Delta t}, v_{h}\right)+\left(b^{n} \nabla \gamma_{h}^{n}, \nabla v_{h}\right)=\left(f^{n}, v_{h}\right), \quad \forall v_{h} \in V_{h}, \\
\left(\gamma_{h}^{n}, g_{h}\right)+\left(\boldsymbol{\sigma}_{h}^{n}, \nabla g_{h}\right)=0, \quad \forall g_{h} \in V_{h}, \\
\left(\boldsymbol{\sigma}_{h}^{n}, \mathbf{z}_{h}\right)+\left(a^{n} \lambda_{h}^{n}, \mathbf{z}_{h}\right)=0, \quad \forall \mathbf{z}_{h} \in \mathbf{W}_{h}, \\
\left(\lambda_{h}^{n}, \mathbf{w}_{h}\right)-\left(\nabla u_{h}^{n}, \mathbf{w}_{h}\right)=0, \quad \forall \mathbf{w}_{h} \in \mathbf{W}_{h} .
\end{gathered}
$$

For deriving the fully discrete error estimates, we now decompose the errors as

$$
\begin{aligned}
& \gamma^{n}-\gamma_{h}^{n}=\gamma^{n}-\mathscr{P}_{h} \gamma^{n}+\mathscr{P}_{h} \gamma^{n}-\gamma_{h}^{n}=\psi^{n}+\omega^{n} ; \\
& u^{n}-u_{h}^{n}=u^{n}-\mathscr{R}_{h} u^{n}+\mathscr{R}_{h} u^{n}-u_{h}^{n}=\eta^{n}+\varsigma^{n} ; \\
& \lambda^{n}-\lambda_{h}^{n}=\lambda^{n}-\mathscr{R}_{h} \lambda^{n}+\mathscr{R}_{h} \lambda^{n}-\lambda_{h}^{n}=\boldsymbol{\delta}^{n}+\boldsymbol{\theta}^{n} ; \\
& \boldsymbol{\sigma}^{n}-\boldsymbol{\sigma}_{h}^{n}=\boldsymbol{\sigma}^{n}-\mathscr{R}_{h} \boldsymbol{\sigma}^{n}+\mathscr{R}_{h} \boldsymbol{\sigma}^{n}-\boldsymbol{\sigma}_{h}^{n}=\boldsymbol{\rho}^{n}+\boldsymbol{\xi}^{n} .
\end{aligned}
$$

We will prove the theorem for the fully discrete error estimates.

Theorem 12. Suppose that $\gamma, \gamma_{t}, u_{t} \in L^{2}\left(H^{2}(\Omega)\right), \lambda, \sigma \in$ $L^{\infty}\left(\left(H^{1}(\Omega)\right)^{2}\right), \gamma \in L^{\infty}\left(H^{2}(\Omega)\right)$, and $u_{t t} \in L^{2}\left(L^{2}(\Omega)\right)$; then there exists a constant $C>0$ independent of the spatial mesh parameter $h$ and the time step $\Delta t$ such that

$$
\begin{aligned}
& \left\|u^{J}-u_{h}^{J}\right\| \leq C h^{2}\left(\|u\|_{L^{\infty}\left(H^{2}\right)}+\|\| \vee \| \mid\right)+C \Delta t\left\|u_{t t}\right\|_{L^{2}\left(L^{2}\right)}, \\
& \left\|u^{J}-u_{h}^{J}\right\|_{1} \leq C h\left(\|u\|_{L^{\infty}\left(H^{2}\right)}+\|\lambda\|_{L^{\infty}\left(\left(H^{1}\right)^{2}\right)}+\|\| \boldsymbol{\nabla} \|\right) \\
& +C \Delta t\left\|u_{t t}\right\|_{L^{2}\left(L^{2}\right)}, \\
& \left\|\lambda^{J}-\lambda_{h}^{J}\right\| \leq C h\left(\|\lambda\|_{L^{\infty}\left(\left(H^{1}\right)^{2}\right)}+\|\|\|\|\right)+C \Delta t\left\|u_{t t}\right\|_{L^{2}\left(L^{2}\right)}, \\
& \left\|\boldsymbol{\sigma}^{J}-\boldsymbol{\sigma}_{h}^{J}\right\| \leq C h\left(\|\boldsymbol{\sigma}\|_{L^{\infty}\left(\left(H^{1}\right)^{2}\right)}+\|\| \boldsymbol{\nabla} \|\right)+C \Delta t\left\|u_{t t}\right\|_{L^{2}\left(L^{2}\right)},
\end{aligned}
$$

Proof. Using (8), (10a), (10b), (10c), (34a), (34b), (34c), (34d), (36a), (36b), (36c), and (36d) at $t=t_{n}$, we obtain the error equations

$$
\begin{aligned}
& \left(\frac{\varsigma^{n}-\varsigma^{n-1}}{\Delta t}, v_{h}\right)+\left(b^{n} \nabla \omega^{n}, \nabla v_{h}\right) \\
& =-\left(\frac{\eta^{n}-\eta^{n-1}}{\Delta t}, v_{h}\right)+\left(R_{1}^{n}, v_{h}\right), \quad \forall v_{h} \in V_{h}, \\
& \left(\omega^{n}, g_{h}\right)+\left(\xi^{n}, \nabla g_{h}\right)=-\left(\psi^{n}, g_{h}\right), \quad \forall g_{h} \in V_{h}, \\
& \left(\xi^{n}, \mathbf{z}_{h}\right)+\left(a^{n} \boldsymbol{\theta}^{n}, \mathbf{z}_{h}\right)=0, \quad \forall \mathbf{z}_{h} \in \mathbf{W}_{h}, \\
& \left(\boldsymbol{\theta}^{n}, \mathbf{w}_{h}\right)-\left(\nabla \varsigma^{n}, \mathbf{w}_{h}\right)=0, \quad \forall \mathbf{w}_{h} \in \mathbf{W}_{h} .
\end{aligned}
$$

Take $v_{h}=\omega^{n}$ in (39a), $g_{h}=\left(\varsigma^{n}-\varsigma^{n-1}\right) / \Delta t$ in (39b), $\mathbf{z}_{h}=$ $\left(\nabla \varsigma^{n}-\nabla \varsigma^{n-1}\right) / \Delta t$ in (39c), and $\mathbf{w}_{h}=\left(\nabla \varsigma^{n}-\nabla \varsigma^{n-1}\right) / \Delta t$ in (39d) to get

$$
\begin{aligned}
& \left\|\left(b^{n}\right)^{1 / 2} \nabla \omega^{n}\right\|^{2} \\
& =-\left(\frac{\varsigma^{n}-\varsigma^{n-1}}{\Delta t}, \omega^{n}\right)-\left(\frac{\eta^{n}-\eta^{n-1}}{\Delta t}, \omega^{n}\right)+\left(R_{1}^{n}, \omega^{n}\right) \\
& =\left(\xi^{n}, \frac{\nabla \varsigma^{n}-\nabla \varsigma^{n-1}}{\Delta t}\right)-\left(\frac{\eta^{n}-\eta^{n-1}}{\Delta t}, \omega^{n}\right)+\left(R_{1}^{n}, \omega^{n}\right) \\
& +\left(\psi^{n}, \frac{\varsigma^{n}-\varsigma^{n-1}}{\Delta t}\right) \\
& =-\left(a^{n} \boldsymbol{\theta}^{n}, \frac{\nabla \varsigma^{n}-\nabla \varsigma^{n-1}}{\Delta t}\right)-\left(\frac{\eta^{n}-\eta^{n-1}}{\Delta t}, \omega^{n}\right) \\
& +\left(R_{1}^{n}, \omega^{n}\right)+\left(\psi^{n}, \frac{\varsigma^{n}-\varsigma^{n-1}}{\Delta t}\right) \\
& =-\left(a^{n} \frac{\nabla \varsigma^{n}-\nabla \varsigma^{n-1}}{\Delta t}, \nabla \varsigma^{n}\right)-\left(\frac{\eta^{n}-\eta^{n-1}}{\Delta t}, \omega^{n}\right) \\
& +\left(R_{1}^{n}, \omega^{n}\right)+\left(\psi^{n}, \frac{\varsigma^{n}-\varsigma^{n-1}}{\Delta t}\right) \\
& =-\frac{1}{2 \Delta t}\left(\left\|\left(a^{n}\right)^{1 / 2} \nabla \varsigma^{n}\right\|^{2}-\left\|\left(a^{n-1}\right)^{1 / 2} \nabla \varsigma^{n-1}\right\|^{2}\right) \\
& -\frac{1}{2 \Delta t}\left\|a^{n}\left(\nabla \varsigma^{n}-\nabla \varsigma^{n-1}\right)\right\|^{2} \\
& +\frac{1}{2}\left(\frac{a^{n}-a^{n-1}}{\Delta t} \nabla \varsigma^{n-1}, \nabla \varsigma^{n-1}\right)-\left(\frac{\eta^{n}-\eta^{n-1}}{\Delta t}, \omega^{n}\right) \\
& +\left(R_{1}^{n}, \omega^{n}\right)+\left(\psi^{n}, \frac{\varsigma^{n}-\varsigma^{n-1}}{\Delta t}\right) \text {. }
\end{aligned}
$$

where $\left|\||\boldsymbol{|}|\| \triangleq\left\|u_{t}\right\|_{L^{2}\left(H^{2}\right)}+\|\gamma\|_{L^{\infty}\left(H^{2}\right)}+\|\gamma\|_{L^{2}\left(H^{2}\right)}+\left\|\gamma_{t}\right\|_{L^{2}\left(H^{2}\right)}\right.$. 
By (40), we have

$$
\begin{aligned}
\left\|\left(b^{n}\right)^{1 / 2} \nabla \omega^{n}\right\|^{2}+\frac{1}{2 \Delta t}\left\|a^{n}\left(\nabla \varsigma^{n}-\nabla \varsigma^{n-1}\right)\right\|^{2} \\
+\frac{1}{2 \Delta t}\left(\left\|\left(a^{n}\right)^{1 / 2} \nabla \varsigma^{n}\right\|^{2}-\left\|\left(a^{n-1}\right)^{1 / 2} \nabla \varsigma^{n-1}\right\|^{2}\right) \\
=\frac{1}{2}\left(\frac{a^{n}-a^{n-1}}{\Delta t} \nabla \varsigma^{n-1}, \nabla \varsigma^{n-1}\right)-\left(\frac{\eta^{n}-\eta^{n-1}}{\Delta t}, \omega^{n}\right) \\
+\left(R_{1}^{n}, \omega^{n}\right)+\left(\psi^{n}, \frac{\varsigma^{n}-\varsigma^{n-1}}{\Delta t}\right) \\
=\frac{1}{2}\left(\frac{1}{\Delta t} \int_{t_{n-1}}^{t_{n}} a_{t}(s) d s \nabla \varsigma^{n-1}, \nabla \varsigma^{n-1}\right)-\left(\frac{\eta^{n}-\eta^{n-1}}{\Delta t}, \omega^{n}\right) \\
+\left(R_{1}^{n}, \omega^{n}\right)+\frac{\left(\psi^{n}, \varsigma^{n}\right)-\left(\psi^{n-1}, \varsigma^{n-1}\right)}{\Delta t} \\
+\left(\varsigma^{n-1}, \frac{\psi^{n}-\psi^{n-1}}{\Delta t}\right) .
\end{aligned}
$$

Multiplying by $2 \Delta t$, summing (41) from $n=1$ to $J$, and using (19), the resulting equation becomes

$$
\begin{aligned}
& a_{0}\left\|\nabla \varsigma^{J}\right\|^{2}+2 b_{0} \Delta t \sum_{n=1}^{J}\left\|\nabla \omega^{n}\right\|^{2} \\
& \leq 2 \Delta t \sum_{n=1}^{J}\left(\left\|\frac{\eta^{n}-\eta^{n-1}}{\Delta t}\right\|+\left\|R_{1}^{n}\right\|\right)\left\|\omega^{n}\right\| \\
& +\sup _{t \in\left[t_{n-1}, t_{n}\right]}\left|a_{t}(t)\right| \Delta t \sum_{n=1}^{J-1}\left\|\nabla \varsigma^{n}\right\|^{2} \\
& +\Delta t \sum_{n=1}^{J}\left(\left\|\varsigma^{n-1}\right\|^{2}+\left\|\frac{\psi^{n}-\psi^{n-1}}{\Delta t}\right\|^{2}\right)+\left\|\psi^{J}\right\|\left\|\varsigma^{J}\right\| \\
& \leq C \Delta t \sum_{n=1}^{J}\left(\left\|\frac{\eta^{n}-\eta^{n-1}}{\Delta t}\right\|^{2}+\left\|R_{1}^{n}\right\|^{2}\right)+b_{0} \Delta t \sum_{n=1}^{J}\left\|\nabla \omega^{n}\right\|^{2} \\
& +\frac{a_{0}}{2}\left\|\nabla \varsigma^{J}\right\|^{2} \cdot \\
& +C \Delta t \sum_{n=1}^{J}\left(\left\|\nabla \varsigma^{n-1}\right\|^{2}+\left\|\frac{\psi^{n}-\psi^{n-1}}{\Delta t}\right\|^{2}\right)+C\left\|\psi^{J}\right\|^{2}
\end{aligned}
$$

Use (42) and Gronwall lemma to get

$$
\begin{aligned}
\left\|\nabla \varsigma^{J}\right\|^{2}+\Delta t \sum_{n=1}^{J}\left\|\nabla \omega^{n}\right\|^{2} \\
\leq C\left\|\psi^{J}\right\|^{2} \\
\quad+C \Delta t \sum_{n=1}^{J}\left(\left\|\frac{\eta^{n}-\eta^{n-1}}{\Delta t}\right\|^{2}+\left\|\frac{\psi^{n}-\psi^{n-1}}{\Delta t}\right\|^{2}+\left\|R_{1}^{n}\right\|^{2}\right) .
\end{aligned}
$$

Note that

$$
\begin{gathered}
\left\|R_{1}^{n}\right\|^{2} \leq C \Delta t \int_{t_{n-1}}^{t_{n}}\left\|u_{t t}\right\|^{2} d s, \\
\left\|\frac{\eta^{n}-\eta^{n-1}}{\Delta t}\right\|^{2} \leq \frac{1}{\Delta t} \int_{t_{n-1}}^{t_{n}}\left\|\eta_{t}(s)\right\|^{2} d s, \\
\left\|\frac{\psi^{n}-\psi^{n-1}}{\Delta t}\right\|^{2} \leq \frac{1}{\Delta t} \int_{t_{n-1}}^{t_{n}}\left\|\psi_{t}(s)\right\|^{2} d s .
\end{gathered}
$$

Substitute (44) into (43) to get

$$
\begin{gathered}
\left\|\nabla \varsigma^{J}\right\|^{2}+\Delta t \sum_{n=1}^{J}\left\|\nabla \omega^{n}\right\|^{2} \\
\leq C\left(\left\|\psi^{J}\right\|^{2}+\int_{t_{0}}^{t_{J}}\left(\left\|\eta_{t}(s)\right\|^{2}+\left\|\psi_{t}(s)\right\|^{2}\right) d s\right. \\
\left.+(\Delta t)^{2} \int_{t_{0}}^{t_{J}}\left\|u_{t t}\right\|^{2} d s\right) .
\end{gathered}
$$

Combining (19) and (45), we obtain

$$
\begin{aligned}
\left\|\zeta^{J}\right\|^{2} & +\Delta t \sum_{n=1}^{J}\left\|\omega^{n}\right\|^{2} \\
\leq & C\left(\left\|\psi^{J}\right\|^{2}+\int_{t_{0}}^{t_{J}}\left(\left\|\eta_{t}(s)\right\|^{2}+\left\|\psi_{t}(s)\right\|^{2}\right) d s\right. \\
& \left.+(\Delta t)^{2} \int_{t_{0}}^{t_{J}}\left\|u_{t t}\right\|^{2} d s\right) .
\end{aligned}
$$

Taking $\mathbf{w}_{h}=\boldsymbol{\theta}^{n}$ in (39d) and $\mathbf{z}_{h}=\boldsymbol{\xi}^{n}$ in (39c) and using (45), we have

$$
\begin{aligned}
\left\|\boldsymbol{\theta}^{J}\right\|^{2} & +\left\|\boldsymbol{\xi}^{J}\right\|^{2} \\
\leq & C\left(\left\|\psi^{J}\right\|^{2}+\int_{t_{0}}^{t_{J}}\left(\left\|\eta_{t}(s)\right\|^{2}+\left\|\psi_{t}(s)\right\|^{2}\right) d s\right. \\
& \left.+(\Delta t)^{2} \int_{t_{0}}^{t_{J}}\left\|u_{t t}\right\|^{2} d s\right) .
\end{aligned}
$$

Combining Lemmas 5-7, (45), (46), and (47) with the triangle inequality, we accomplish the proof for Theorem 12.

In the following analysis, we will derive the optimal a priori error estimates in $L^{2}$ and $H^{1}$-norm for $\gamma$. 
TABLE 1: $L^{2}$ and $H^{1}$-norms and convergence order for $u$.

\begin{tabular}{|c|c|c|c|c|}
\hline$(h, \Delta t)$ & $\left\|u-u_{h}\right\|_{L^{\infty}\left(L^{2}(\Omega)\right)}$ & Order & $\left\|u-u_{h}\right\|_{L^{\infty}\left(H^{1}(\Omega)\right)}$ & Order \\
\hline$(\sqrt{2} / 8,1 / 8)$ & $2.8933 e-002$ & & $2.3766 e-001$ & \\
\hline$(\sqrt{2} / 16,1 / 16)$ & $8.2158 e-003$ & 1.8162 & $1.2986 e-001$ & 0.8719 \\
\hline$(\sqrt{2} / 32,1 / 32)$ & $2.1012 e-003$ & 1.9672 & $6.8466 e-002$ & 0.9235 \\
\hline$(\sqrt{2} / 64,1 / 64)$ & $5.3511 e-004$ & 1.9733 & $3.5245 e-002$ & 0.9580 \\
\hline
\end{tabular}

TABLE 2: $L^{2}$ and $H^{1}$-norms and convergence order for $\gamma$.

\begin{tabular}{lccc}
\hline$(h, \Delta t)$ & $\left\|\gamma-\gamma_{h}\right\|_{L^{\infty}\left(L^{2}(\Omega)\right)}$ & Order & $\left\|\gamma-\gamma_{h}\right\|_{L^{\infty}\left(H^{1}(\Omega)\right)}$ \\
\hline$(\sqrt{2} / 8,1 / 8)$ & $3.4238 e-001$ & & $4.5457 e+000$ \\
$(\sqrt{2} / 16,1 / 16)$ & $9.2701 e-002$ & 1.8849 & $2.5429 e+000$ \\
$(\sqrt{2} / 32,1 / 32)$ & $2.3840 e-002$ & 1.9592 & $1.3493 e+000$ \\
$(\sqrt{2} / 64,1 / 64)$ & $5.8649 e-003$ & 2.0232 & $6.9558 e-001$ \\
\hline
\end{tabular}

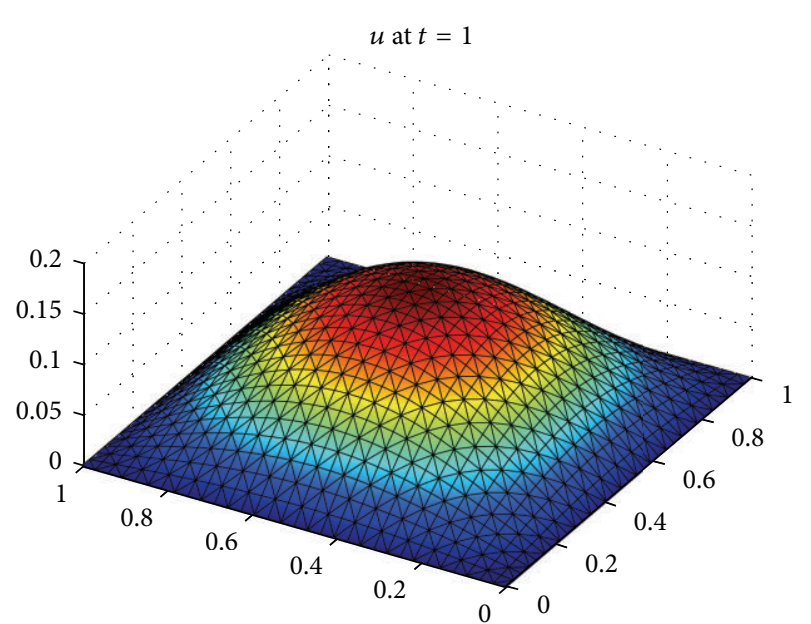

FIGURE 1: Surface for exact solution $u$.

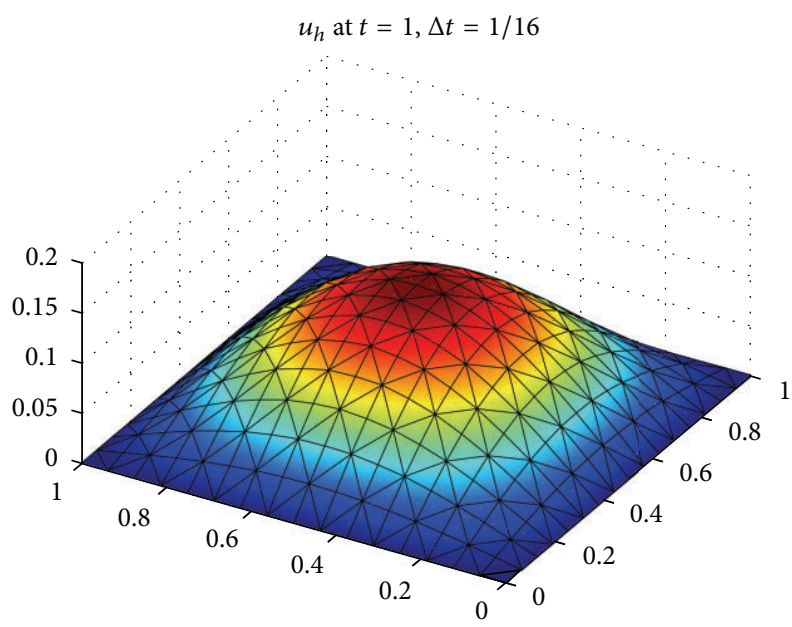

FIGURE 2: Surface for numerical solution $u_{h}$.
Theorem 13. Suppose that $\gamma, \gamma_{t}, u_{t} \in L^{2}\left(H^{2}(\Omega)\right), \partial^{2} u / \partial t^{2}$, $\partial^{4} u / \partial t^{4} \in L^{2}\left(L^{2}(\Omega)\right), \gamma \in L^{\infty}\left(H^{2}(\Omega)\right)$, and $\partial^{3} u / \partial t^{3} \in$ $L^{\infty}\left(L^{2}(\Omega)\right)$; then there exists a constant $C>0$ independent of the spatial mesh parameter $h$ and time discretization parameter $\Delta t$ such that

$$
\begin{aligned}
&\left\|\gamma^{J}-\gamma_{h}^{J}\right\|_{j} \leq C h^{2-j}(\|\gamma\|_{L^{\infty}\left(H^{2}\right)}+\|\gamma\|_{L^{2}\left(H^{2}\right)} \\
&\left.+\left\|u_{t}\right\|_{L^{2}\left(H^{2}\right)}+\left\|\gamma_{t}\right\|_{L^{2}\left(H^{2}\right)}\right) \\
&+\Delta t\left(\left\|\frac{\partial^{2} u}{\partial t^{2}}\right\|_{L^{2}\left(L^{2}\right)}+\left\|\frac{\partial^{3} u}{\partial t^{3}}\right\|_{L^{\infty}\left(L^{2}\right)}\right. \\
&\left.+\left\|\frac{\partial^{4} u}{\partial t^{4}}\right\|_{L^{2}\left(L^{2}\right)}\right), \quad j=0,1 .
\end{aligned}
$$

Proof. Use (39a)-(39d) to get

$$
\begin{aligned}
& \left(\frac{\varsigma^{n}-\varsigma^{n-1}}{\Delta t}, v_{h}\right)+\left(b^{n} \nabla \omega^{n}, \nabla v_{h}\right) \\
& =-\left(\frac{\eta^{n}-\eta^{n-1}}{\Delta t}, v_{h}\right)+\left(R_{1}^{n}, v_{h}\right), \quad \forall v_{h} \in V_{h}, \\
& \left(\frac{\omega^{n}-\omega^{n-1}}{\Delta t}, g_{h}\right)+\left(\frac{\xi^{n}-\xi^{n-1}}{\Delta t}, \nabla g_{h}\right) \\
& =-\left(\frac{\psi^{n}-\psi^{n-1}}{\Delta t}, g_{h}\right), \quad \forall g_{h} \in V_{h}, \\
& \left(\frac{\xi^{n}-\xi^{n-1}}{\Delta t}, \mathbf{z}_{h}\right)+\left(\frac{a^{n} \boldsymbol{\theta}^{n}-a^{n-1} \boldsymbol{\theta}^{n-1}}{\Delta t}, \mathbf{z}_{h}\right) \\
& =0, \quad \forall \mathbf{z}_{h} \in \mathbf{W}_{h}, \\
& \left(\frac{\boldsymbol{\theta}^{n}-\boldsymbol{\theta}^{n-1}}{\Delta t}, \mathbf{w}_{h}\right)-\left(\frac{\nabla \varsigma^{n}-\nabla \varsigma^{n-1}}{\Delta t}, \mathbf{w}_{h}\right) \\
& =0, \quad \forall \mathbf{w}_{h} \in \mathbf{W}_{h} .
\end{aligned}
$$


$\sigma_{1}$ (left) and $\sigma_{2}$ (right) at $t=1$
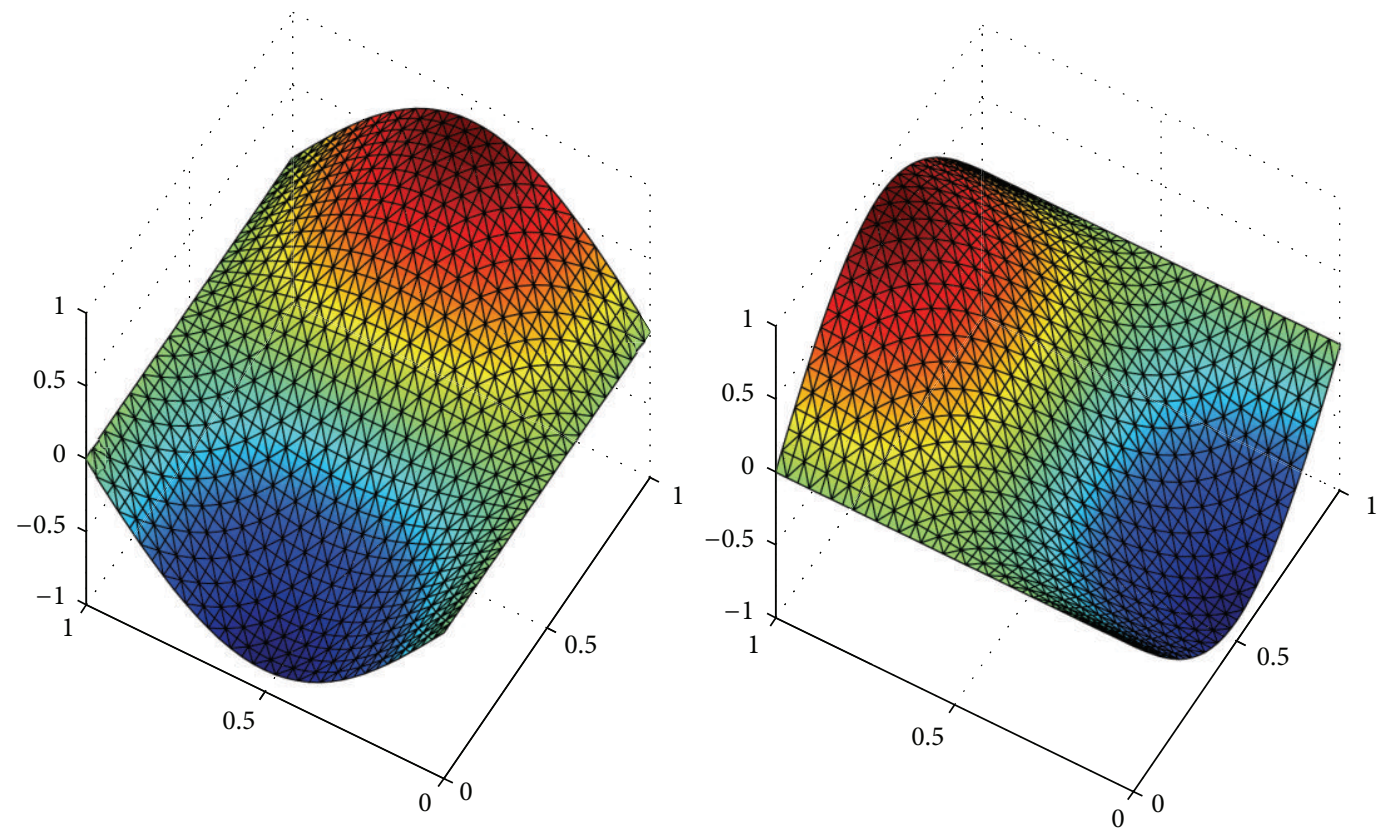

FIGURE 3: Surface for exact solution $\boldsymbol{\sigma}=\left(\boldsymbol{\sigma}_{1}, \boldsymbol{\sigma}_{2}\right)$.

$\sigma_{1 h}$ (left) and $\sigma_{2 h}$ (right) at $t=1, \Delta t=1 / 16$
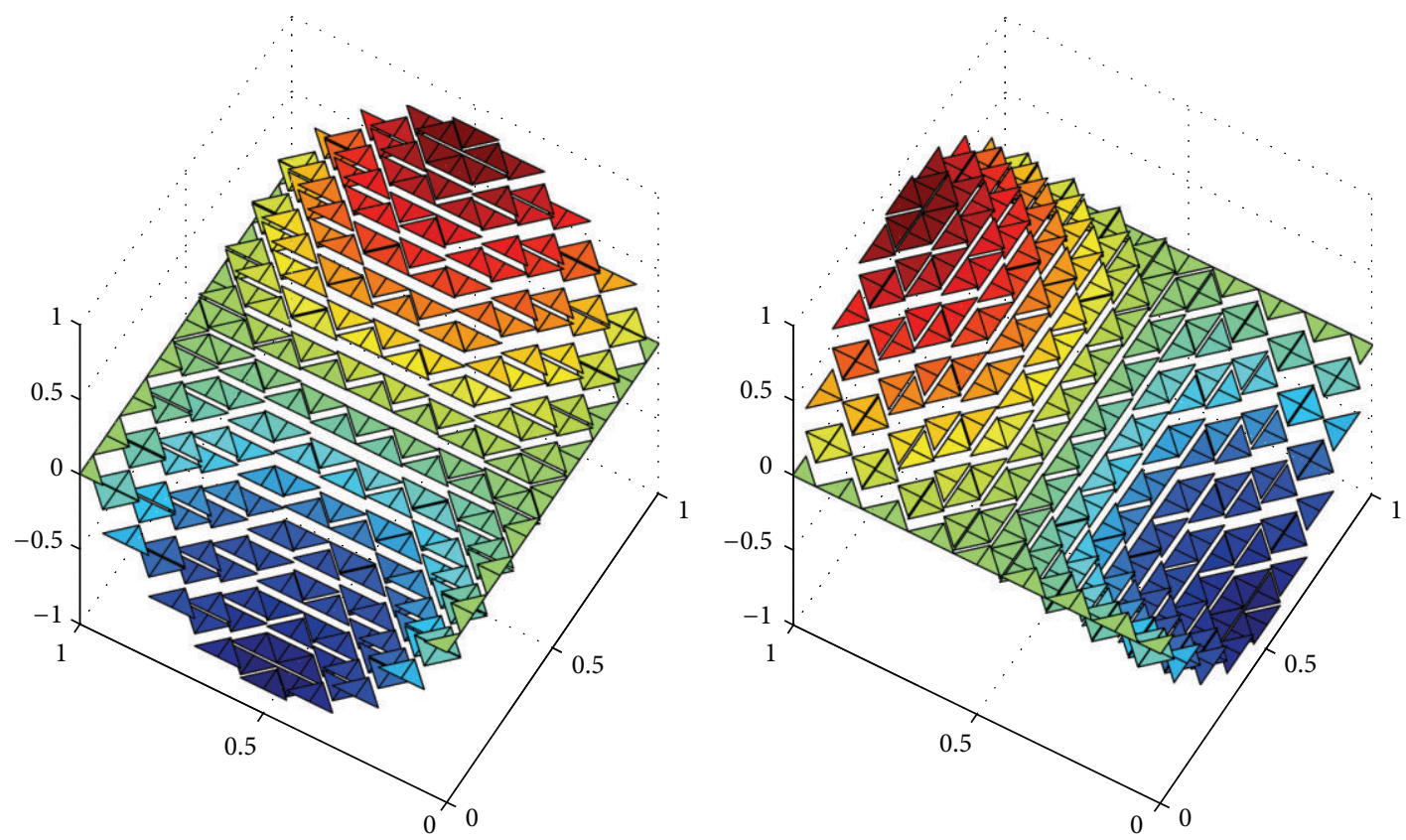

FIGURE 4: Surface for numerical solution $\sigma_{h}=\left(\sigma_{1 h}, \sigma_{2 h}\right)$.

Taking $v_{h}=\left(\omega^{n}-\omega^{n-1}\right) / \Delta t, g_{h}=\left(\varsigma^{n}-\varsigma^{n-1}\right) / \Delta t, \mathbf{z}_{h}=\left(\nabla \varsigma^{n}-\right.$ $\left.\nabla \varsigma^{n-1}\right) / \Delta t$, and $\mathbf{w}_{h}=\left(\nabla \varsigma^{n}-\nabla \varsigma^{n-1}\right) / \Delta t$, respectively, in (49a)(49d), we get

$$
\begin{gathered}
\frac{1}{2 \Delta t}\left(\left\|\left(b^{n}\right)^{1 / 2} \nabla \omega^{n}\right\|^{2}-\left\|\left(b^{n-1}\right)^{1 / 2} \nabla \omega^{n-1}\right\|^{2}\right) \\
+\frac{1}{2 \Delta t}\left\|b^{n}\left(\nabla \omega^{n}-\nabla \omega^{n-1}\right)\right\|^{2}
\end{gathered}
$$

$$
\begin{aligned}
= & \left(b^{n} \nabla \omega^{n}, \nabla \frac{\omega^{n}-\omega^{n-1}}{\Delta t}\right) \\
& +\frac{1}{2}\left(\frac{b^{n}-b^{n-1}}{\Delta t} \nabla \omega^{n-1}, \nabla \omega^{n-1}\right) \\
= & -\left(\frac{\varsigma^{n}-\varsigma^{n-1}}{\Delta t}, \frac{\omega^{n}-\omega^{n-1}}{\Delta t}\right)
\end{aligned}
$$


$\lambda_{1}$ (left) and $\boldsymbol{\lambda}_{2}$ (right) at $t=1$
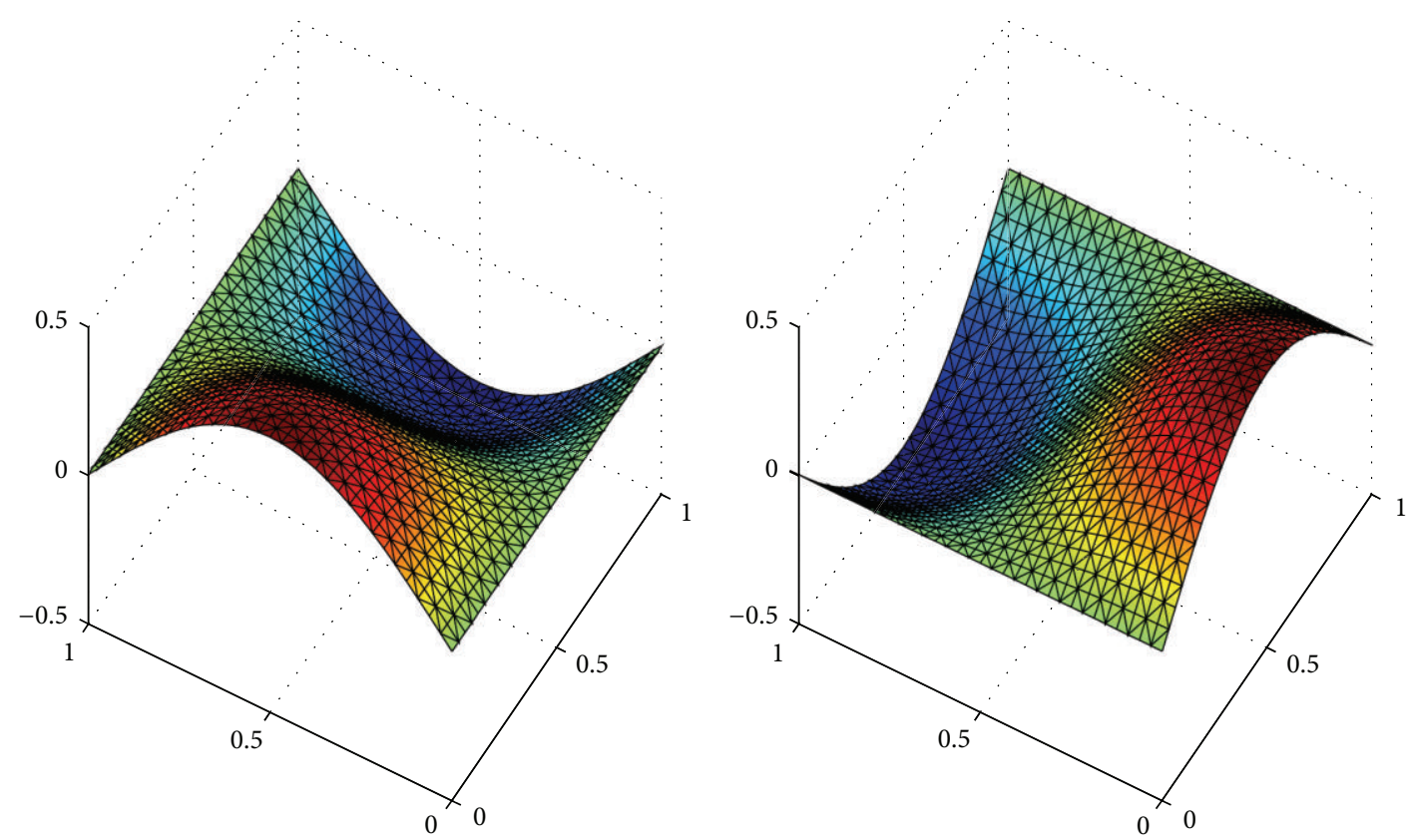

FIGURE 5: Surface for exact solution $\lambda=\left(\lambda_{1}, \lambda_{2}\right)$.

TABLE 3: $\left(L^{2}\right)^{2}$-norm and convergence order for $\lambda$ and $\sigma$.

\begin{tabular}{|c|c|c|c|c|}
\hline$(h, \Delta t)$ & $\left\|\boldsymbol{\sigma}-\boldsymbol{\sigma}_{h}\right\|_{L^{\infty}\left(\left(L^{2}(\Omega)\right)^{2}\right)}$ & Order & $\left\|\boldsymbol{\lambda}-\boldsymbol{\lambda}_{h}\right\|_{L^{\infty}\left(\left(L^{2}(\Omega)\right)^{2}\right)}$ & Order \\
\hline$(\sqrt{2} / 8,1 / 8)$ & $2.3958 e-001$ & & $2.3589 e-001$ & \\
\hline$(\sqrt{2} / 16,1 / 16)$ & $1.3011 e-001$ & 0.8808 & $1.2960 e-001$ & 0.8641 \\
\hline$(\sqrt{2} / 32,1 / 32)$ & $6.8501 e-002$ & 0.9255 & $6.8434 e-002$ & 0.9213 \\
\hline$(\sqrt{2} / 64,1 / 64)$ & $3.5250 e-002$ & 0.9585 & $3.5242 e-002$ & 0.9574 \\
\hline
\end{tabular}

$$
\begin{aligned}
& -\left(\frac{\eta^{n}-\eta^{n-1}}{\Delta t}, \frac{\omega^{n}-\omega^{n-1}}{\Delta t}\right) \\
& +\left(R_{1}^{n}, \frac{\omega^{n}-\omega^{n-1}}{\Delta t}\right)-\left(\frac{\eta^{n}-\eta^{n-1}}{\Delta t}, \frac{\omega^{n}-\omega^{n-1}}{\Delta t}\right) \\
& +\left(R_{1}^{n}, \frac{\omega^{n}-\omega^{n-1}}{\Delta t}\right)+\frac{1}{2}\left(\frac{b^{n}-b^{n-1}}{\Delta t} \nabla \omega^{n-1}, \nabla \omega^{n-1}\right) \\
& +\frac{1}{2}\left(\frac{b^{n}-b^{n-1}}{\Delta t} \nabla \omega^{n-1}, \nabla \omega^{n-1}\right) \\
& =\left(\frac{\xi^{n}-\xi^{n-1}}{\Delta t}, \frac{\nabla \varsigma^{n}-\nabla \varsigma^{n-1}}{\Delta t}\right)+\left(\frac{\psi^{n}-\psi^{n-1}}{\Delta t}, \frac{\varsigma^{n}-\varsigma^{n-1}}{\Delta t}\right) \\
& =-\left\|\left(a^{n}\right)^{1 / 2} \frac{\nabla \varsigma^{n}-\nabla \varsigma^{n-1}}{\Delta t}\right\|^{2} \\
& +\left(R_{1}^{n}, \frac{\omega^{n}-\omega^{n-1}}{\Delta t}\right)-\left(\frac{\eta^{n}-\eta^{n-1}}{\Delta t}, \frac{\omega^{n}-\omega^{n-1}}{\Delta t}\right) \\
& -\left(\frac{a^{n}-a^{n-1}}{\Delta t} \boldsymbol{\theta}^{n-1}, \frac{\nabla \varsigma^{n}-\nabla \varsigma^{n-1}}{\Delta t}\right) \\
& +\frac{1}{2}\left(\frac{b^{n}-b^{n-1}}{\Delta t} \nabla \omega^{n-1}, \nabla \omega^{n-1}\right) \\
& +\left(\frac{\psi^{n}-\psi^{n-1}}{\Delta t}, \frac{\varsigma^{n}-\varsigma^{n-1}}{\Delta t}\right) \\
& =-\left(\frac{a^{n}\left(\boldsymbol{\theta}^{n}-\boldsymbol{\theta}^{n-1}\right)}{\Delta t}, \frac{\nabla \varsigma^{n}-\nabla \varsigma^{n-1}}{\Delta t}\right) \\
& -\left(\frac{a^{n}-a^{n-1}}{\Delta t} \boldsymbol{\theta}^{n-1}, \frac{\nabla \varsigma^{n}-\nabla \varsigma^{n-1}}{\Delta t}\right) \\
& +\left(\frac{\psi^{n}-\psi^{n-1}}{\Delta t}, \frac{\varsigma^{n}-\varsigma^{n-1}}{\Delta t}\right) \\
& +\left(R_{1}^{n}, \frac{\omega^{n}-\omega^{n-1}}{\Delta t}\right) \\
& -\left(\frac{\eta^{n}-\eta^{n-1}}{\Delta t}, \frac{\omega^{n}-\omega^{n-1}}{\Delta t}\right) \\
& +\frac{1}{2}\left(\frac{b^{n}-b^{n-1}}{\Delta t} \nabla \omega^{n-1}, \nabla \omega^{n-1}\right) \text {. }
\end{aligned}
$$



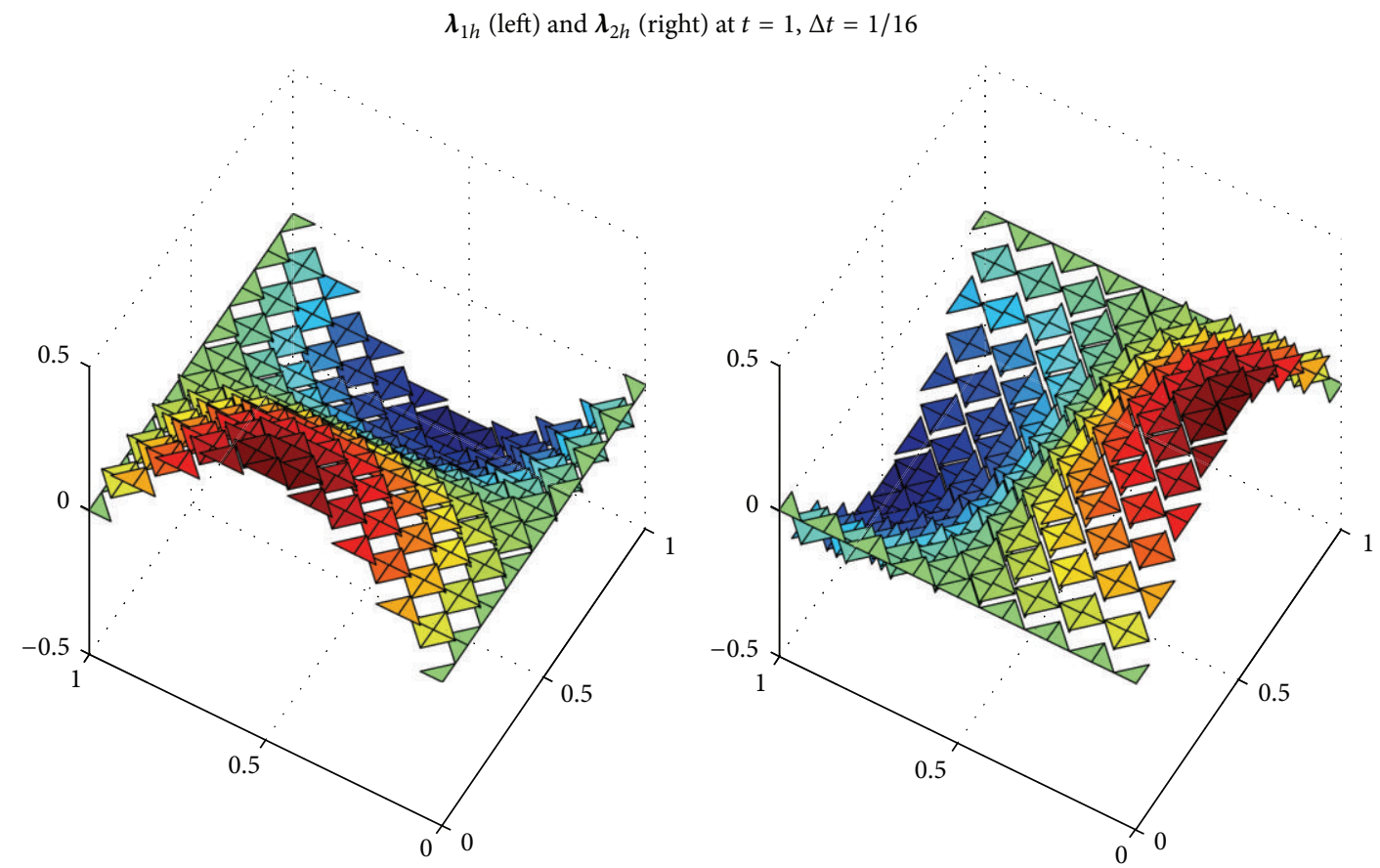

FIgURE 6: Surface for numerical solution $\lambda_{h}=\left(\lambda_{1 h}, \lambda_{2 h}\right)$.

By using (50), we obtain

$$
\begin{aligned}
& \frac{1}{2 \Delta t}\left(\left\|\left(b^{n}\right)^{1 / 2} \nabla \omega^{n}\right\|^{2}-\left\|\left(b^{n-1}\right)^{1 / 2} \nabla \omega^{n-1}\right\|^{2}\right) \\
& +\frac{1}{2 \Delta t}\left\|b^{n}\left(\nabla \omega^{n}-\nabla \omega^{n-1}\right)\right\|^{2} \\
& +\left\|\left(a^{n}\right)^{1 / 2} \frac{\nabla \varsigma^{n}-\nabla \varsigma^{n-1}}{\Delta t}\right\|^{2} \\
& =-\left(\frac{a^{n}-a^{n-1}}{\Delta t} \boldsymbol{\theta}^{n-1}, \nabla \frac{\varsigma^{n}-\varsigma^{n-1}}{\Delta t}\right) \\
& +\left(\frac{\psi^{n}-\psi^{n-1}}{\Delta t}, \frac{\varsigma^{n}-\varsigma^{n-1}}{\Delta t}\right) \\
& +\frac{\left(\omega^{n}, R_{1}^{n}\right)-\left(\omega^{n-1}, R_{1}^{n-1}\right)}{\Delta t} \\
& -\left(\frac{R_{1}^{n}-R_{1}^{n-1}}{\Delta t}, \omega^{n-1}\right) \\
& -\frac{\left(\left(\eta^{n}-\eta^{n-1}\right) / \Delta t, \omega^{n}\right)-\left(\left(\eta^{n-1}-\eta^{n-2}\right) / \Delta t, \omega^{n-1}\right)}{\Delta t} \\
& +\left(\frac{\eta^{n}-2 \eta^{n-1}+\eta^{n-2}}{(\Delta t)^{2}}, \omega^{n-1}\right) \\
& +\frac{1}{2}\left(\frac{b^{n}-b^{n-1}}{\Delta t} \nabla \omega^{n-1}, \nabla \omega^{n-1}\right) .
\end{aligned}
$$

Multiply (51) by $2 \Delta t$, sum from $n=1$ to $J$, and use (19) to get

$$
\begin{aligned}
& b_{0}\left\|\nabla \omega^{J}\right\|^{2}+2 a_{0} \Delta t \sum_{n=1}^{J}\left\|\frac{\nabla \varsigma^{n}-\nabla \varsigma^{n-1}}{\Delta t}\right\|^{2} \\
& \leq 2 \Delta t \sum_{n=1}^{J}\left(\left\|\frac{a^{n}-a^{n-1}}{\Delta t} \boldsymbol{\theta}^{n-1}\right\|\left\|\frac{\nabla \varsigma^{n}-\nabla \varsigma^{n-1}}{\Delta t}\right\|\right. \\
& \left.+\left\|\frac{\psi^{n}-\psi^{n-1}}{\Delta t}\right\|\left\|\frac{\varsigma^{n}-\varsigma^{n-1}}{\Delta t}\right\|\right) \\
& +2\left\|\omega^{J}\right\|\left\|R_{1}^{J}\right\|+2 \Delta t \sum_{n=1}^{J}\left\|\frac{R_{1}^{n}-R_{1}^{n-1}}{\Delta t}\right\|\left\|\omega^{n-1}\right\| \\
& +2\left\|\frac{\eta^{J}-\eta^{J-1}}{\Delta t} c\right\|\left\|\omega^{J}\right\| \\
& +2 \Delta t \sum_{n=1}^{J}\left\|\frac{\eta^{n}-2 \eta^{n-1}+\eta^{n-2}}{(\Delta t)^{2}}\right\|\left\|\omega^{n-1}\right\| \\
& +C \Delta t \sum_{n=1}^{J}\left\|\nabla \omega^{n-1}\right\|^{2} \\
& \leq C \Delta t \sum_{n=1}^{J}\left(\left\|\boldsymbol{\theta}^{n-1}\right\|+\left\|\frac{\psi^{n}-\psi^{n-1}}{\Delta t}\right\|^{2}\right. \\
& \left.+\left\|\frac{\eta^{n}-2 \eta^{n-1}+\eta^{n-2}}{(\Delta t)^{2}}\right\|^{2}+\left\|\frac{R_{1}^{n}-R_{1}^{n-1}}{\Delta t}\right\|^{2}\right) \\
& +a_{0} \Delta t \sum_{n=1}^{J}\left\|\frac{\nabla \varsigma^{n}-\nabla \varsigma^{n-1}}{\Delta t}\right\|^{2}
\end{aligned}
$$




$$
\begin{aligned}
& +C\left(\left\|R_{1}^{J}\right\|^{2}+\left\|\frac{\eta^{J}-\eta^{J-1}}{\Delta t}\right\|^{2}\right)+\frac{b_{0}}{2}\left\|\nabla \omega^{J}\right\|^{2} \\
& +C \Delta t \sum_{n=1}^{J}\left\|\nabla \omega^{n-1}\right\|^{2} .
\end{aligned}
$$

Use Gronwall lemma to obtain

$$
\begin{aligned}
&\left\|\nabla \omega^{J}\right\|^{2} \leq C \Delta t \sum_{n=1}^{J}\left(\left\|\boldsymbol{\theta}^{n-1}\right\|+\left\|\frac{\psi^{n}-\psi^{n-1}}{\Delta t}\right\|^{2}\right. \\
&\left.+\left\|\frac{R_{1}^{n}-R_{1}^{n-1}}{\Delta t}\right\|^{2}+\left\|\frac{\eta^{n}-2 \eta^{n-1}+\eta^{n-2}}{(\Delta t)^{2}}\right\|^{2}\right) \\
&+C\left(\left\|R_{1}^{J}\right\|^{2}+\left\|\frac{\eta^{J}-\eta^{J-1}}{\Delta t}\right\|^{2}\right) .
\end{aligned}
$$

Note that

$$
\begin{aligned}
\left\|\frac{R_{1}^{n}-R_{1}^{n-1}}{\Delta t}\right\|^{2} & =\left\|\frac{u^{n}-2 u^{n-1}+u^{n-2}}{(\Delta t)^{2}}-\frac{u_{t}\left(t_{n}\right)-u_{t}\left(t_{n-1}\right)}{\Delta t}\right\|^{2} \\
& =\left\|\frac{u^{n}-2 u^{n-1}+u^{n-2}}{(\Delta t)^{2}}-u_{t t}\left(t_{\Delta_{*}}\right)\right\|^{2} \\
\leq & C\left[\Delta t \int_{t_{n-2}}^{t_{n}}\left\|\frac{\partial^{4} u}{\partial t^{4}}\right\| d s+\Delta t\left\|\frac{\partial^{3} u}{\partial t^{3}}\right\|_{L^{\infty}\left(L^{2}\right)}\right]^{2} \\
\leq & C(\Delta t)^{2} \int_{t_{n-2}}^{t_{n}}\left\|\frac{\partial^{4} u}{\partial t^{4}}\right\|^{2} d s+(\Delta t)^{2}\left\|\frac{\partial^{3} u}{\partial t^{3}}\right\|_{L^{\infty}\left(L^{2}\right)}^{2} \\
& \text { where } t_{\Delta_{*}} \in\left(t_{n-1}, t_{n}\right) .
\end{aligned}
$$

Substitute (44), (47) and (54) into (53) to obtain

$$
\begin{gathered}
\left\|\nabla \omega^{J}\right\|^{2} \leq C\left(\|\psi\|_{L^{\infty}\left(L^{2}\right)}^{2}+\int_{t_{0}}^{t_{J}}\left(\left\|\eta_{t}(s)\right\|^{2}+\left\|\psi_{t}(s)\right\|^{2}\right) d s\right. \\
+(\Delta t)^{2}\left[\left\|\frac{\partial^{3} u}{\partial t^{3}}\right\|_{L^{\infty}\left(L^{2}\right)}^{2}+\left\|\frac{\partial^{2} u}{\partial t^{2}}\right\|_{L^{2}\left(L^{2}\right)}^{2}\right. \\
\left.\left.+\left\|\frac{\partial^{4} u}{\partial t^{4}}\right\|_{L^{2}\left(L^{2}\right)}^{2}\right]\right) .
\end{gathered}
$$

Use (55) and Poincaré inequality (19) to get

$$
\begin{gathered}
\left\|\omega^{J}\right\|^{2} \leq C\left(\|\psi\|_{L^{\infty}\left(L^{2}\right)}^{2}+\int_{t_{0}}^{t_{J}}\left(\left\|\eta_{t}(s)\right\|^{2}+\left\|\psi_{t}(s)\right\|^{2}\right) d s\right. \\
+(\Delta t)^{2}\left[\left\|\frac{\partial^{3} u}{\partial t^{3}}\right\|_{L^{\infty}\left(L^{2}\right)}^{2}+\left\|\frac{\partial^{2} u}{\partial t^{2}}\right\|_{L^{2}\left(L^{2}\right)}^{2}\right. \\
\left.\left.+\left\|\frac{\partial^{4} u}{\partial t^{4}}\right\|_{L^{2}\left(L^{2}\right)}^{2}\right]\right) .
\end{gathered}
$$

Combining Lemmas 6-7, (55), (56) and using the triangle inequality, we complete the proof.

\section{Numerical Results}

In this section, we would like to give some numerical results for the coupling method of EMFE method and FE method proposed and analyzed in this paper. We consider the following initial-boundary value problem of fourth-order parabolic system:

$$
\begin{gathered}
u_{t}+\nabla \cdot(b \nabla(\nabla \cdot(a(t) \nabla u)))=f(\mathbf{x}, t), \quad(\mathbf{x}, t) \in \Omega \times J, \\
u(\mathbf{x}, t)=\Delta u(\mathbf{x}, t)=0, \quad(\mathbf{x}, t) \in \partial \Omega \times \bar{J}, \\
u(\mathbf{x}, 0)=\sin \left(\pi x_{1}\right) \sin \left(\pi x_{2}\right), \quad \mathbf{x}=\left(x_{1}, x_{2}\right) \in \Omega,
\end{gathered}
$$

where $\Omega=[0,1] \times[0,1], J=(0,1], a(t)=1+t^{2}, b=1$, and $f(\mathbf{x}, t)$ is chosen so that the exact solution for the scalar unknown function is

$$
u(\mathbf{x}, t)=e^{-2 t} \sin \left(\pi x_{1}\right) \sin \left(\pi x_{2}\right),
$$

its exact gradient is

$$
\lambda=\nabla u=e^{-2 t} \pi\left[\begin{array}{l}
\cos \left(\pi x_{1}\right) \sin \left(\pi x_{2}\right) \\
\sin \left(\pi x_{1}\right) \cos \left(\pi x_{2}\right)
\end{array}\right],
$$

its exact flux function is

$$
\boldsymbol{\sigma}=-a \boldsymbol{\lambda}=e^{-2 t}\left(1+t^{2}\right) \pi\left[\begin{array}{l}
\cos \left(\pi x_{1}\right) \sin \left(\pi x_{2}\right) \\
\sin \left(\pi x_{1}\right) \cos \left(\pi x_{2}\right)
\end{array}\right],
$$

and the diffusion term is

$$
\gamma=\nabla \cdot \boldsymbol{\sigma}=2 \pi^{2} e^{-2 t}\left(1+t^{2}\right) \sin \left(\pi x_{1}\right) \sin \left(\pi x_{2}\right) .
$$

Dividing the domain $\Omega$ into the triangulations of mesh size $h$ uniformly and choosing the piecewise linear space $V_{h}$ with index $k=1$ and $h=\sqrt{2} \Delta t=\sqrt{2} / 8, \sqrt{2} / 16, \sqrt{2} / 32$, $\sqrt{2} / 64$, we obtain the optimal a priori error estimates in $L^{2}$ and $H^{1}$-norm for the scalar unknown $u$ in Table 1. Similarly, from Table 2, we can find that both $\left\|\gamma-\gamma_{h}\right\|_{L^{\infty}\left(H^{1}(\Omega)\right)}$ and 


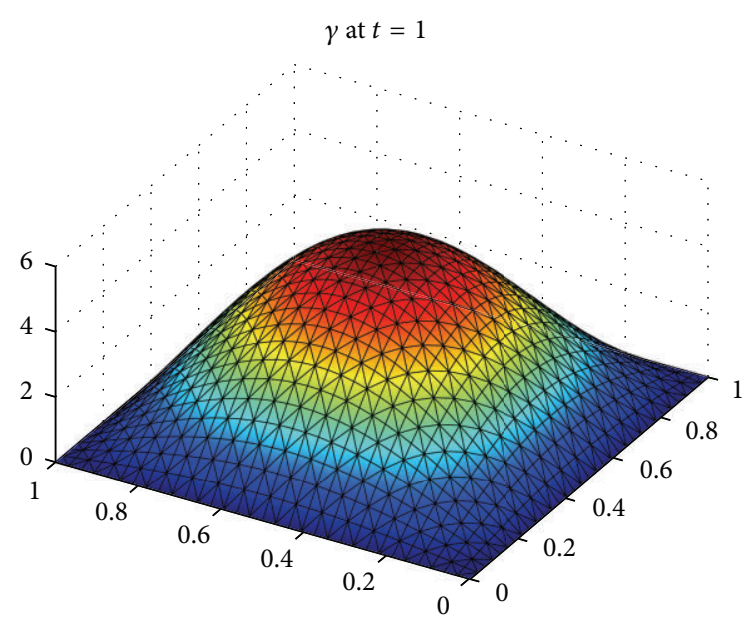

FIGURE 7: Surface for exact solution $\gamma$.

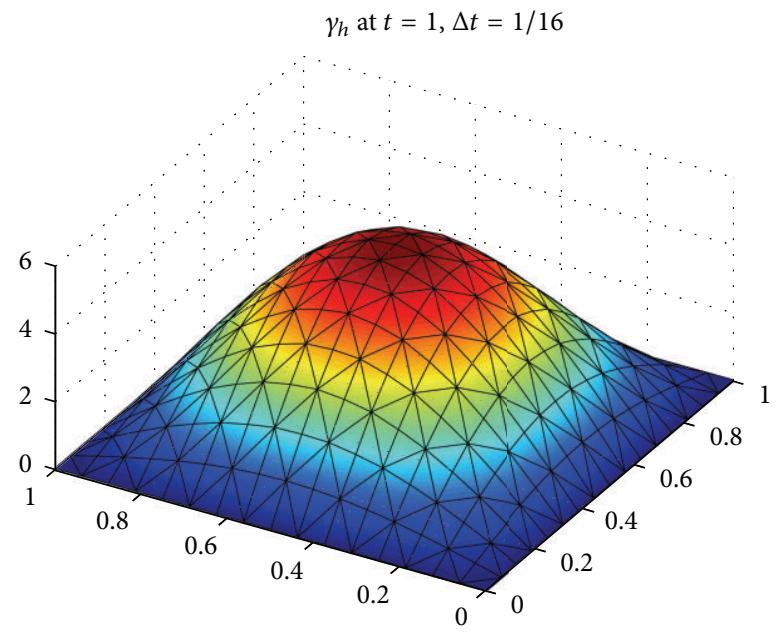

FIGURE 8: Surface for numerical solution $\gamma_{h}$.

$\left\|\gamma-\gamma_{h}\right\|_{L^{\infty}\left(L^{2}(\Omega)\right)}$ for the diffusion term $\gamma$ are optimal, too. At the same time, we take the corresponding piecewise constant space $\mathbf{W}_{h}$ with index $k=0$ for the gradient $\lambda$ and the flux $\sigma$ and obtain some convergence results in $\left(L^{2}\right)^{2}$-norm with $h=\sqrt{2} \Delta t=\sqrt{2} / 8, \sqrt{2} / 16, \sqrt{2} / 32, \sqrt{2} / 64$ in Table 3.

Taking $t=1$ with $h=\sqrt{2} \Delta t=\sqrt{2} / 32$, we show the surfaces of the exact solution $u, \sigma, \lambda$, and $\gamma$ in Figures 1, 3, 5, and 7, respectively. Figures $2,4,6$, and 8 show the surfaces of the numerical solution $u_{h}, \sigma_{h}, \lambda_{h}$ and $\gamma_{h}$ at $t=1$ with $h=\sqrt{2} \Delta t=\sqrt{2} / 16$, respectively.

From the convergence results in Tables 1-3 and Figures 18 , we can find that the numerical results verify our theoretical analysis.

\section{Concluding Remarks}

In this article, we study a new coupling method of new EMFE scheme [29] and FE scheme for fourth-order partial differential equation of parabolic type. The new EMFE method's gradient belongs to the simple square integrable
$\left(L^{2}(\Omega)\right)^{2}$ space; therefore, the regularity requirement on the gradient solution $\lambda=\nabla u$ is reduced. We obtain the optimal priori error estimates in $L^{2}$ and $H^{1}$-norm for both the scalar unknown $u$ and the diffusion term $\gamma$ and the priori error estimates in $\left(L^{2}\right)^{2}$-norm for its gradient $\lambda$ and its flux $\sigma$. Finally, a numerical example is provided to verify the efficiency of our methods. At the same time, we apply the new coupling method based on new EMFE method and FE method to solving the fourth-order eigenvalue problems, which will be shown in another article.

In the near future, we will study the new expanded mixed finite element method for other partial differential equations, such as fourth-order wave equations. Moreover, we will apply some new techniques based on the combination of two-grid methods [36, 37] and new (expanded) mixed methods [29$32,38]$ for solving fourth-order nonlinear elliptic equation and fourth-order nonlinear parabolic equations.

\section{Acknowledgments}

The authors thank the referees and editor for their valuable suggestions and comments, which greatly improve the paper. This work is supported by National Natural Science Fund (11061021), Natural Science Fund of Inner Mongolia Autonomous Region (2012MS0108, 2012MS0106), Scientific Research Projection of Higher Schools of Inner Mongolia (NJZZ12011, NJ10006, NJZY13199), and Program of Higherlevel talents of Inner Mongolia University (125119, 30105125132).

\section{References}

[1] Z. Chen, "Analysis of expanded mixed methods for fourth-order elliptic problems," Numerical Methods for Partial Differential Equations, vol. 13, no. 5, pp. 483-503, 1997.

[2] S. Chen, M. Liu, and Z. Qiao, "An anisotropic nonconforming element for fourth order elliptic singular perturbation problem," International Journal of Numerical Analysis and Modeling, vol. 7, no. 4, pp. 766-784, 2010.

[3] J. Li, "Full-order convergence of a mixed finite element method for fourth-order elliptic equations," Journal of Mathematical Analysis and Applications, vol. 230, no. 2, pp. 329-349, 1999.

[4] J. Li, "Optimal error estimates of mixed finite element methods for a fourth-order nonlinear elliptic problem," Journal of Mathematical Analysis and Applications, vol. 334, no. 1, pp. 183-195, 2007.

[5] J. Li, "Optimal convergence analysis of mixed finite element methods for fourth-order elliptic and parabolic problems," Numerical Methods for Partial Differential Equations, vol. 22, no. 4, pp. 884-896, 2006.

[6] J. Li, "Mixed methods for fourth-order elliptic and parabolic problems using radial basis functions," Advances in Computational Mathematics, vol. 23, no. 1-2, pp. 21-30, 2005.

[7] T. Zhang, "Finite element analysis for the Cahn-Hilliard equation," Mathematica Numerica Sinica, vol. 28, no. 3, pp. 281-292, 2006.

[8] Y. Liu, H. Li, S. He, W. Gao, and Z. C. Fang, " $H^{1}$-galerkin mixed element method and numerical simulation for the fourth-order parabolic partial differential equations," Mathematica Numerica Sinica, vol. 34, no. 2, pp. 259-274, 2012. 
[9] J. W. Barrett, J. F. Blowey, and H. Garcke, "Finite element approximation of a fourth order nonlinear degenerate parabolic equation," Numerische Mathematik, vol. 80, no. 4, pp. 525-556, 1998.

[10] S. He, H. Li, and Y. Liu, "Analysis of mixed finite element methods for fourth-order wave equations," Computers \& Mathematics with Applications, vol. 65, no. 1, pp. 1-16, 2013.

[11] Y. Liu, H. Li, S. He, W. Gao, and Z. C. Fang, "A $C^{1}$-Conforming finite element method for nonlinear fourth-order hyperbolic equation," World Academy of Science, Engineering and Technology, vol. 80, pp. 1423-1427, 2011.

[12] P. Danumjaya and A. K. Pani, "Mixed finite element methods for a fourth order reaction diffusion equation," Numerical Methods for Partial Differential Equations, vol. 28, no. 4, pp. 1227-1251, 2012.

[13] D. Y. Shi and Y. C. Peng, "Finite element methods for fourthorder eigenvalue problems on anisotropic meshes," Chinese Journal of Engineering Mathematics, vol. 25, no. 6, pp. 10291034, 2008.

[14] Z. X. Chen, Expanded Mixed Finite Element Methods for Linear Second Order Elliptic Problems I, vol. 1219 of IMA preprint series, Institute for Mathematics and Its Applications, University of Minnesota, Minneapolis, Minn, USA, 1994.

[15] Z. Chen, "Expanded mixed finite element methods for linear second-order elliptic problems. I," RAIRO Modélisation Mathématique et Analyse Numérique, vol. 32, no. 4, pp. 479-499, 1998.

[16] Z. Chen, "Expanded mixed finite element methods for quasilinear second order elliptic problems. II," RAIRO Modélisation Mathématique et Analyse Numérique, vol. 32, no. 4, pp. 501-520, 1998.

[17] T. Arbogast, M. F. Wheeler, and I. Yotov, "Mixed finite elements for elliptic problems with tensor coefficients as cell-centered finite differences," SIAM Journal on Numerical Analysis, vol. 34, no. 2, pp. 828-852, 1997.

[18] C. S. Woodward and C. N. Dawson, "Analysis of expanded mixed finite element methods for a nonlinear parabolic equation modeling flow into variably saturated porous media," SIAM Journal on Numerical Analysis, vol. 37, no. 3, pp. 701-724, 2000.

[19] Y. Chen, Y. Huang, and D. Yu, "A two-grid method for expanded mixed finite-element solution of semilinear reaction-diffusion equations," International Journal for Numerical Methods in Engineering, vol. 57, no. 2, pp. 193-209, 2003.

[20] Y. Chen, H.-W. Liu, and S. Liu, "Analysis of two-grid methods for reaction-diffusion equations by expanded mixed finite element methods," International Journal for Numerical Methods in Engineering, vol. 69, no. 2, pp. 408-422, 2007.

[21] Y. Chen, P. Luan, and Z. Lu, "Analysis of two-grid methods for nonlinear parabolic equations by expanded mixed finite element methods," Advances in Applied Mathematics and Mechanics, vol. 1, no. 6, pp. 830-844, 2009.

[22] W. Liu, H. X. Rui, and H. Guo, "A two-grid method with expanded mixed element for nonlinear reaction-diffusion equations," Acta Mathematicae Applicatae Sinica, vol. 27, no. 3, pp. 495-502, 2011.

[23] H. Z. Chen and H. Wang, "An optimal-order error estimate on an $H^{1}$-Galerkin mixed method for a nonlinear parabolic equation in porous medium flow," Numerical Methods for Partial Differential Equations, vol. 26, no. 1, pp. 188-205, 2010.

[24] Y. Liu, Analysis and numerical simulation of nonstandard mixed element methods [Ph.D. thesis], Inner Mongolia University, Hohhot, China, 2011.
[25] H. Che, Y. Wang, and Z. Zhou, "An optimal error estimates of $H^{1}$-Galerkin expanded mixed finite element methods for nonlinear viscoelasticity-type equation," Mathematical Problems in Engineering, vol. 2011, Article ID 570980, 18 pages, 2011.

[26] H. Rui and T. Lu, "An expanded mixed covolume method for elliptic problems," Numerical Methods for Partial Differential Equations, vol. 21, no. 1, pp. 8-23, 2005.

[27] D. Kim and E. J. Park, "A posteriori error estimator for expanded mixed hybrid methods," Numerical Methods for Partial Differential Equations, vol. 23, no. 2, pp. 330-349, 2007.

[28] Y. Liu, H. Li, J. Wang, and W. Gao, "A new positive definite expanded mixed finite element method for parabolic integrodifferential equations," Journal of Applied Mathematics, vol. 2012, Article ID 391372, 24 pages, 2012.

[29] Y. Liu, H. Li, et al., "A new expanded mixed finite element method for elliptic equations," http://nmg.zsksedu.com/3267/ 2012011690642.htm.

[30] S. C. Chen and H. R. Chen, "New mixed element schemes for a second-order elliptic problem," Mathematica Numerica Sinica, vol. 32, no. 2, pp. 213-218, 2010.

[31] F. Shi, J. Yu, and K. Li, "A new stabilized mixed finiteelement method for Poisson equation based on two local Gauss integrations for linear element pair," International Journal of Computer Mathematics, vol. 88, no. 11, pp. 2293-2305, 2011.

[32] D. Shi and Y. Zhang, "High accuracy analysis of a new nonconforming mixed finite element scheme for Sobolev equations," Applied Mathematics and Computation, vol. 218, no. 7, pp. 31763186, 2011.

[33] P. G. Ciarlet, The Finite Element Method for Elliptic Problems, North-Holland, Amsterdam, The Netherlands, 1978.

[34] Z. D. Luo, Mixed Finite Element Methods and Applications, Chinese Science Press, Beijing, China, 2006.

[35] D. Zwillinger, Handbook of Differential Equations, Academic Press, Boston, Mass, USA, 3rd edition, 1997.

[36] J. Xu, "Two-grid discretization techniques for linear and nonlinear PDEs," SIAM Journal on Numerical Analysis, vol. 33, no. 5, pp. 1759-1777, 1996.

[37] J. Xu, "A novel two-grid method for semilinear elliptic equations," SIAM Journal on Scientific Computing, vol. 15, no. 1, pp. 231-237, 1994.

[38] Z. Weng, X. Feng, and P. Huang, "A new mixed finite element method based on the Crank-Nicolson scheme for the parabolic problems," Applied Mathematical Modelling, vol. 36, no. 10, pp. 5068-5079, 2012. 


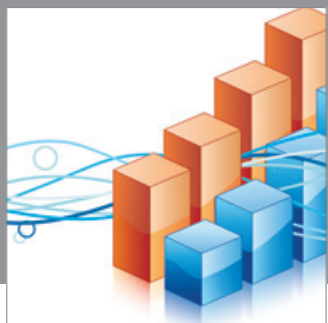

Advances in

Operations Research

mansans

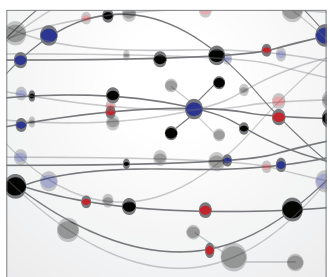

The Scientific World Journal
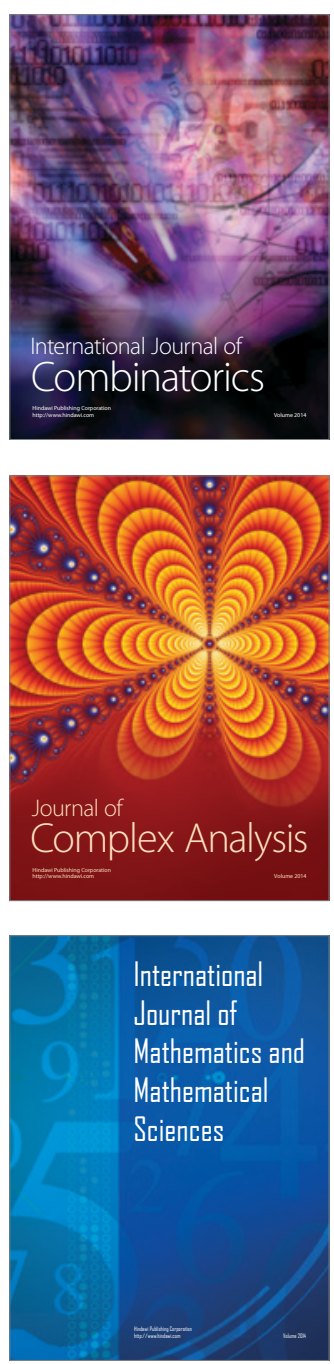
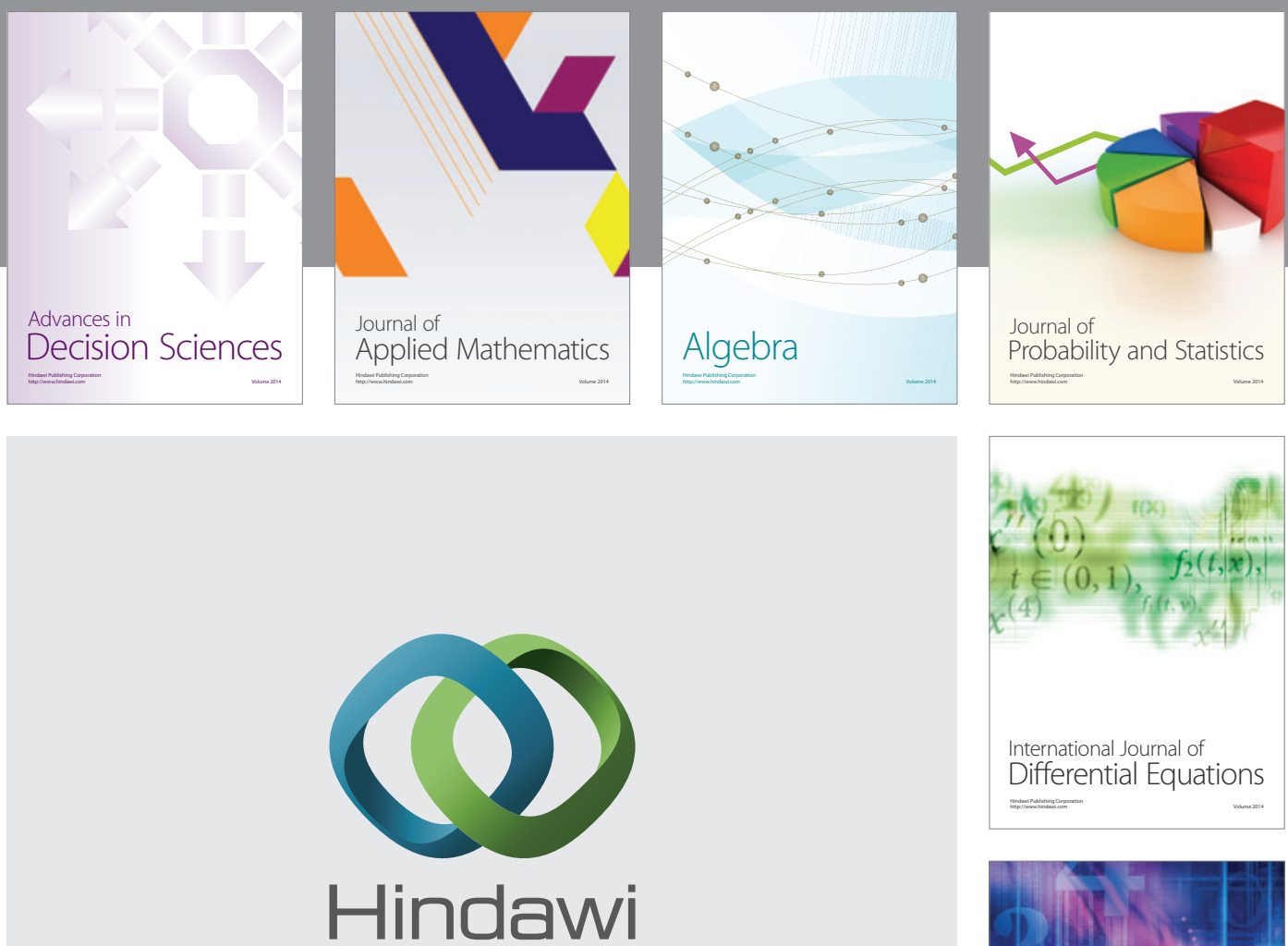

Submit your manuscripts at http://www.hindawi.com
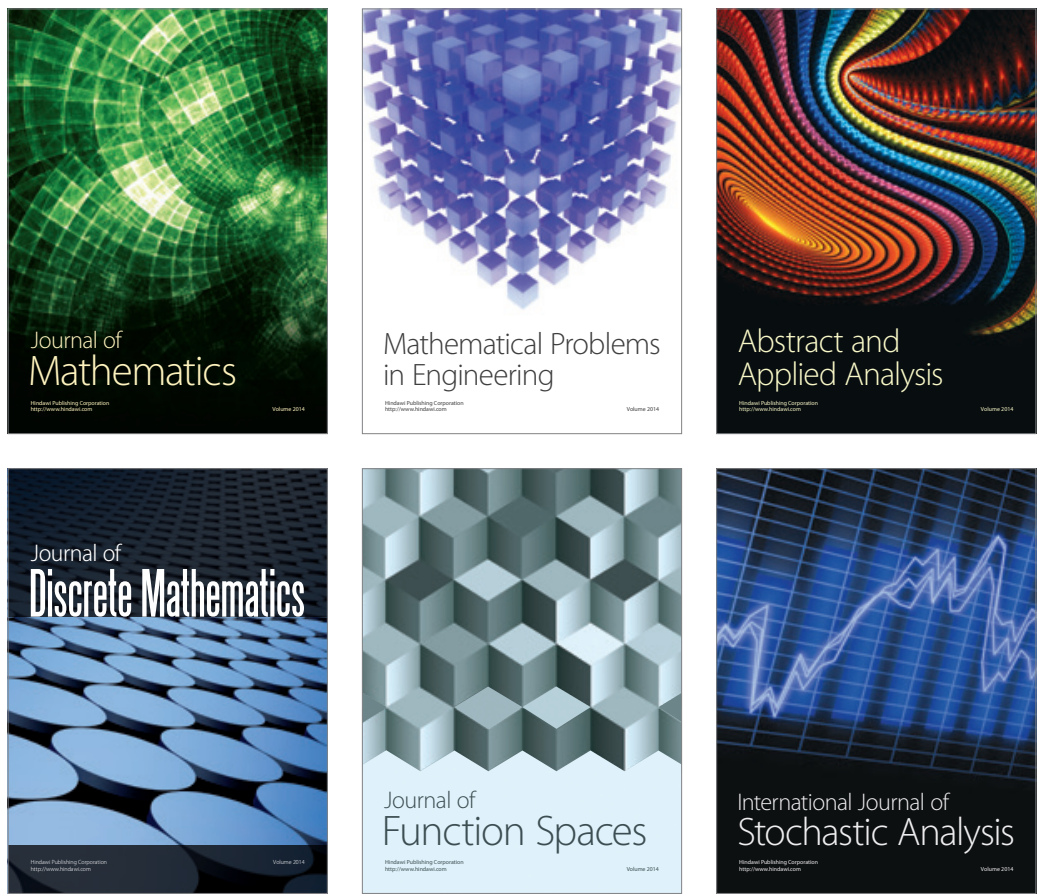

Journal of

Function Spaces

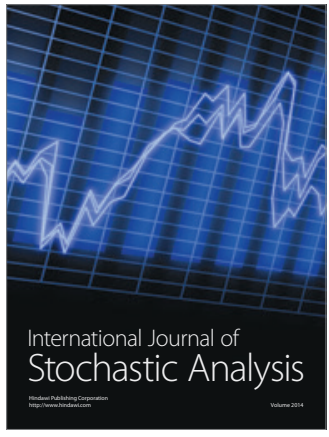

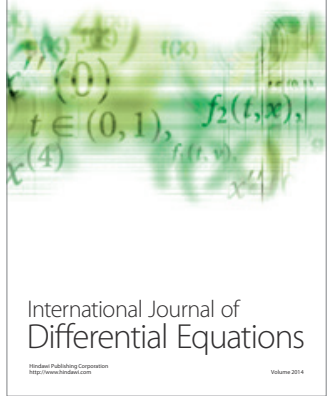
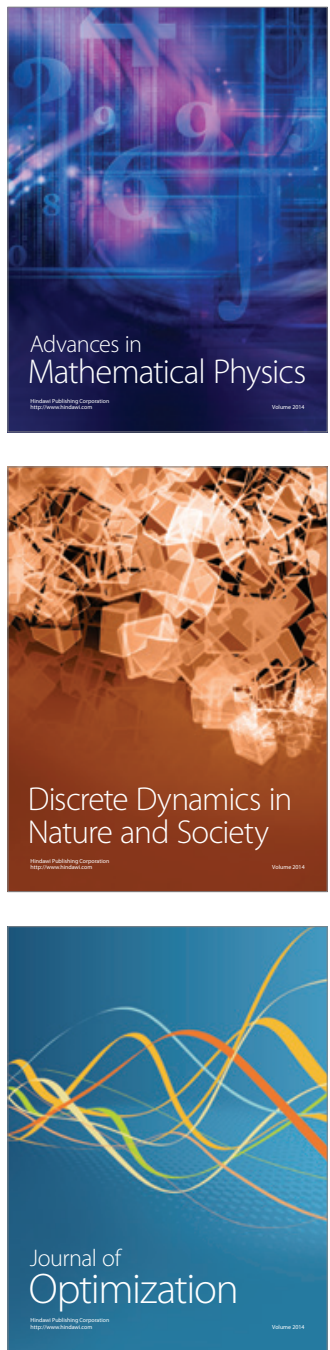